\title{
Nested Hybrid Cylindrical Array Design and DoA Estimation for Massive IoT Networks
}

\author{
Zhipeng Lin, Tiejun Lv, Senior Member, IEEE, Wei Ni, Senior Member, IEEE, J. Andrew Zhang, \\ Senior Member, IEEE, and Ren Ping Liu, Senior Member, IEEE
}

\begin{abstract}
Reducing cost and power consumption while maintaining high network access capability is a key physical-layer requirement of massive Internet of Things (mIoT) networks. Deploying a hybrid array is a cost- and energy-efficient way to meet the requirement, but would penalize system degree of freedom (DoF) and channel estimation accuracy. This is because signals from multiple antennas are combined by a radio frequency (RF) network of the hybrid array. This paper presents a novel hybrid uniform circular cylindrical array (UCyA) for mIoT networks. We design a nested hybrid beamforming structure based on sparse array techniques and propose the corresponding channel estimation method based on the second-order channel statistics. As a result, only a small number of RF chains are required to preserve the DoF of the UCyA. We also propose a new tensor-based two-dimensional (2-D) direction-of-arrival (DoA) estimation algorithm tailored for the proposed hybrid array. The algorithm suppresses the noise components in all tensor modes and operates on the signal data model directly, hence improving estimation accuracy with an affordable computational complexity. Corroborated by a Cramér-Rao lower bound (CRLB) analysis, simulation results show that the proposed hybrid UCyA array and the DoA estimation algorithm can accurately estimate the 2-D DoAs of a large number of IoT devices.
\end{abstract}

Index Terms-Massive IoT, massive MIMO, hybrid beamformer, sparse array, tensor.

\section{INTRODUCTION}

Low-cost, low-power millimeter-wave (mmWave) techniques have been developed to provide radio access capacity for massive Internet of Things (mIoT) applications, such as smart city infrastructure, healthcare and self-driving cars [1][3]. In an mIoT network, a large number of IoT devices are connected to an Internet-enabled system [4], [5]. Combined with advanced multiple access techniques, mmWave massive multiple-input multiple-output (MIMO) can significantly increase network capacity and can be potentially applied to mIoT networks [6]. However, high hardware cost and power consumption are two major obstacles of applying mmWave massive MIMO into mIoT networks [5]. It is unrealistic to

This work was supported in part by the National Natural Science Foundation of China (NSFC) under Grants 61671072, and in part by the Beijing Natural Science Foundation under Grants L192025. (Corresponding author: Tiejun Lv.)

Z. Lin and T. Lv are with the School of Information and Communication Engineering, BUPT, Beijing, China (email: \{linlzp, lvtiejun $\} @$ bupt.edu.cn). Z. Lin is also with the School of Electrical and Data Engineering, UTS, Sydney, Australia.

W. $\mathrm{Ni}$ is with the Data61, CSIRO, Sydney, Australia (e-mail: Wei.Ni@data61.csiro.au).

J. A. Zhang and R. P. Liu are with the School of Electrical and Data Engineering, UTS, Sydney, Australia (e-mail: \{Andrew.Zhang, RenPing.Liu\}@uts.edu.au). provide a radio-frequency $(\mathrm{RF})$ chain for each antenna, as fully digital beamforming techniques would require [7]. Hybrid beamforming is an appropriate architecture in which a lowdimensional digital beamforming in the baseband and a highdimensional analog beamforming at the RF front-end are used [8], [9]. Most conventional channel estimation schemes for hybrid beamforming have been designed with given channel information [9]. Some of them apply RF networks to directly combine the received signals from multiple antennas, resulting in resolution losses of channel estimation accuracy [10], [11]. As a result, the system degree of freedom (DoF), measuring the number of targets which can be sensed and estimated at the base station (BS) [12], would decrease.

To increase the system DoF with a limited number of antennas, the concept of sparse array, such as minimum redundancy array (MRA) [13], minimum hole array (MHA) [14], nested array [12], and coprime array [15], has attracted considerable attention. By exploiting the second-order statistics of impinging signals, these sparse arrays are capable of identifying $O\left(N^{2}\right)$ uncorrelated sources with only $N$ physical elements. However, existing sparse array techniques have been typically used to design linear or square arrays. Compared to square arrays, circular arrays have a much more compact size, less sensitivity to mutual coupling, and inherently more symmetric structure [16], and hence, they are more suitable for mIoT applications.

Channel estimation is challenging for sparse arrays. There have been attempts to apply the celebrated multiple signal classification (MUSIC) algorithm to networks equipped with sparse arrays [12], [15], [17]-[19]. But the estimation accuracy of this algorithm is unsatisfactory, depending on the searching step and signal correlation. Tensor-based multi-dimensional MUSIC algorithms were proposed in [20], [21] for sparse arrays to improve estimation accuracy. However, since the MUSIC spectrum of their algorithms is a product of multiple separable second-order spectra, undesirable cross-terms [21] would arise, leading to incorrect spectral peak search results. To solve this problem, CANDECOMP/PARAFAC (CP)-based tensor channel estimation algorithms were proposed [22]. However, these algorithms have a very high computational complexity.

This paper proposes a new nested massive hybrid uniform circular cylindrical array (UCyA) design and the corresponding tensor-based angle estimation algorithm for the uplink of mIoT networks. By exploiting the sparse array techniques, the proposed hybrid antenna array enables the BS to estimate the 
DoAs of a large number of devices with much fewer RF chains than antennas. As a result, the massive access requirement of mIoT can be met, with significantly reduced hardware cost and network overhead. Authors of [23] and [24] proposed nested sparse circular arrays for direction-of-arrival (DoA) estimation. However, they directly computed the autocorrelation of impinging signals, which unfortunately destructed the original symmetric structures of circular arrays and penalized the channel estimation accuracy significantly. Different from [23] and [24], we transform the nonlinear phase of the UCyA steering vectors to be linear to the element locations, so that the horizontal symmetric structure of UCyA can be preserved. In addition, since the DoA estimation algorithms developed in [23] and [24] were matrix-based, the estimation accuracy gap between the algorithms and the CRLB is large and the algorithms cannot be directly applied to high-dimension DoA estimation. For our new hybrid UCyA array design, we propose a new tensor $n$-rank enhancement method and a new tensor-based two-dimensional (2-D) DoA estimation algorithm. The algorithm suppresses the noise components in each mode of the signal tensor model. As a result, the DoAs of a large number of IoT devices can be accurately estimated with a much smaller number of RF chains. The key contributions of this paper are summarized as follows.

- We design a new nested hybrid UCyA, which reduces the required number of RF chains while preserving the inherently horizontal symmetric structure of the UCyA to maintain a good channel estimation accuracy. The theory of phase-space transformation is first used to transform the nonlinear phase of the UCyA steering vectors to be linear to the element locations. Then, we design the RFchain connection network by exploiting the sparse array technique, and utilize its generated difference coarray for parameter estimation.

- We analyze the rank relationship between signal matrix and the signal tensor model in each dimension, and propose a tensor $n$-rank enhancement method which ensures that the signal and noise subspaces can be properly decomposed in all dimensions.

- We propose a new tensor-based two-dimensional (2-D) DoA estimation algorithm, based on our hybrid array design. We combine the tensor tool with the estimation of signal parameters via rotational invariance technique (ESPRIT) to estimate the elevation angles. Then, we substitute the estimates to derive the azimuth angles by using tensor MUSIC. Simulation results show that, by suppressing the noise components in all tensor modes, the proposed algorithm can significantly improve the estimation accuracy, as compared to the state of the art.

The rest of this paper is organized as follows. The system model is introduced in Sections II. In Sections III and IV, we design the hybrid beamformer and propose the new spatial smoothing-based method to enhance the $n$-rank of measurement tensor. In Section V, we design a new tensor-based 2-D DoA estimation algorithm, and analyze the system complexity.
In Section VII, simulation results are presented, followed by conclusions in Section VIII.

Preliminary and notation: We provide a brief introduction of tensor and the notations used in this paper. Tensor is the generalization of scalar (which has a zero-order mode), vector (which has an one-order mode), and matrix (which has twoorder modes) to arrays which have an arbitrary order of modes. We use $\mathcal{A} \in \mathbb{C}^{I_{1} \times I_{2} \times \cdots \times I_{N}}$ to denote an order- $N$ tensor, whose elements (entries) are $a_{i_{1}, i_{2}, \cdots, i_{N}}, i_{n}=1,2, \ldots, I_{n}$, and the indices in the $n$-th mode of $\mathcal{A}$ range from 1 to $I_{n}$.

In this paper, we use the following notations and operations in accordance with [25].

- $a, \mathbf{a}$ and $\mathbf{A}$ stand for a scalar, a column vector, and a matrix, respectively; $\mathbf{I}_{K}$ and $\mathbf{0}_{M \times K}$ denote a $K \times K$ identity matrix and an $M \times K$ zero matrix, respectively; $\mathbf{A}^{*}, \mathbf{A}^{T}$ and $\mathbf{A}^{H}$ denote the conjugate, transpose and conjugate transpose of $\mathbf{A}$, respectively; $\|\mathbf{A}\|_{\mathrm{F}}$ denotes the Frobenius norm of $\mathbf{A} ; \otimes$ and $\diamond$ denote the Kronecker product and Khatri-Rao product, respectively; and invec $(\cdot)$ denotes the inverse algorithm of vectorization.

- The mode- $n$ unfolding (also known as matricization) of a tensor $\mathcal{A} \in \mathbb{C}^{I_{1} \times I_{2} \times \cdots \times I_{N}}$, denoted by $\mathbf{A}_{(n)} \in$ $\mathbb{C}^{I_{n} \times\left(I_{1} I_{2} \cdots I_{N} / I_{n}\right)}$, arranges the fibers in the $n$-th mode of $\mathcal{A}$ as the columns of the resulting matrix $\mathbf{A}_{(n)}$. The $n$-rank of $\mathcal{Y}$, denoted by $\operatorname{Rank}_{n}(\mathcal{A})$, is the rank of the mode- $n$ unfolding of tensor $\mathcal{A}$.

- The $n$-mode product of a tensor $\mathcal{A} \in \mathbb{C}^{I_{1} \times I_{2} \times \cdots \times I_{N}}$ and a matrix $\mathbf{B} \in \mathbb{C}^{J_{n} \times I_{n}}$ is defined as

$$
\mathcal{C}=\mathcal{A} \times{ }_{n} \mathbf{B} \in \mathbb{C}^{I_{1} \times \cdots \times I_{n-1} \times J_{n} \times I_{n+1} \times \cdots \times I_{N}},
$$

which can be written in the form of the mode- $n$ matricized tensor: $\mathbf{C}_{(n)}=\mathbf{B} \mathbf{A}_{(n)}$.

- The multilinear product of a tensor $\mathcal{A} \in \mathbb{C}^{I_{1} \times I_{2} \times \cdots \times I_{N}}$ and matrices $\mathbf{B}^{(n)} \in \mathbb{C}^{J_{n} \times I_{n}}, n=1,2, \ldots, N$, is a sequence of contractions with each being an $n$-mode product, i.e.,

$\mathcal{C}=\mathcal{A} \times{ }_{1} \mathbf{B}^{(1)} \times{ }_{2} \mathbf{B}^{(2)} \cdots \times{ }_{N} \mathbf{B}^{(N)} \in \mathbb{C}^{J_{1} \times J_{2} \times \cdots \times J_{N}}$,

which can be equivalently expressed as $\mathcal{C}=$ $\llbracket \mathcal{A} ; \mathbf{B}^{(1)}, \mathbf{B}^{(2)}, \ldots, \mathbf{B}^{(N)} \rrbracket$. The mode- $n$ unfolding (or matricization) of $\mathcal{C}$ is given by

$$
\begin{aligned}
\mathbf{C}_{(n)} & =\mathbf{B}^{(n)} \mathbf{A}_{(n)}\left(\mathbf{B}^{(n+1)} \otimes \mathbf{B}^{(n+2)} \otimes\right. \\
& \left.\cdots \otimes \mathbf{B}^{(N)} \otimes \mathbf{B}^{(1)} \otimes \mathbf{B}^{(2)} \otimes \cdots \otimes \mathbf{B}^{(n-1)}\right)^{T}
\end{aligned}
$$

- The outer product of two tensors $\mathcal{A} \in \mathbb{C}^{I_{1} \times I_{2} \times \cdots \times I_{N}}$ and $\mathcal{B} \in \mathbb{C}^{J_{1} \times J_{2} \times \cdots \times J_{M}}$ is given by

$$
\mathcal{C}=\mathcal{A} \circ \mathcal{B} \in \mathbb{C}^{I_{1} \times I_{2} \times \cdots \times I_{N} \times J_{1} \times J_{2} \times \cdots \times J_{M}},
$$

whose elements are $c_{i_{1}, i_{2}, \cdots, i_{N}, j_{1}, j_{2}, \cdots, j_{M}}=a_{i_{1}, i_{2}, \cdots, i_{N}}$. $b_{j_{1}, j_{2}, \cdots, j_{M}}$.

- Two tensors, $\mathcal{A} \in \mathbb{C}^{I_{1} \times I_{2} \times \cdots \times I_{N}}$ and $\mathcal{B} \in$ $\mathbb{C}^{I_{1} \times \cdots \times I_{n-1} \times J_{n} \times I_{n+1} \times \cdots \times I_{N}}$, can be concatenated in their $n$-th mode, as given by

$$
\mathcal{C}=\left[\mathcal{A} \sqcup_{n} \mathcal{B}\right] \in \mathbb{C}^{I_{1} \times \cdots \times I_{n-1} \times\left(I_{n}+J_{n}\right) \times I_{n+1} \times \cdots \times I_{N}} .
$$




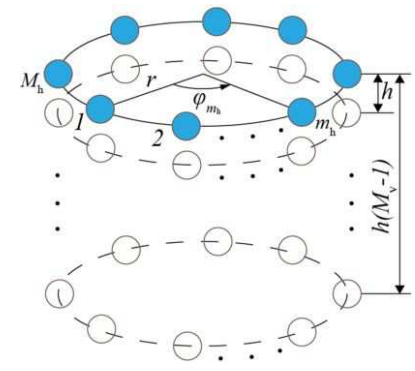

Fig. 1. The geometric model of the UCyA.

- The Tucker decomposition decomposes a tensor $\mathcal{A} \in \mathbb{C}^{I_{1} \times I_{2} \times \cdots \times I_{N}}$ into a core tensor $\mathcal{G} \in \mathbb{C}^{R_{1} \times R_{2} \times \cdots \times R_{N}}$ multiplied by a factor matrix $\mathbf{C}^{(n)}=\left[\mathbf{c}_{r_{n}=1}^{(n)}, \mathbf{c}_{r_{n}=2}^{(n)}, \ldots, \mathbf{c}_{r_{n}=R_{n}}^{(n)}\right] \in \mathbb{C}^{I_{n} \times R_{n}}$ $\left(\mathbf{c}_{r_{n}}^{(n)} \in \mathbb{C}^{I_{n} \times 1}\right.$ and $\left.n=1,2, \ldots, N\right)$ in each mode, i.e.,

$$
\begin{aligned}
\mathcal{A} & =\sum_{r_{1}=1}^{R_{1}} \sum_{r_{2}=1}^{R_{2}} \cdots \sum_{r_{N}=1}^{R_{N}} g_{r_{1} r_{2} \cdots r_{N}}\left(\mathbf{c}_{r_{1}}^{(1)} \circ \mathbf{c}_{r_{2}}^{(2)} \circ \cdots \mathbf{c}_{r_{N}}^{(N)}\right) \\
& =\llbracket \mathcal{G} ; \mathbf{C}^{(1)}, \mathbf{C}^{(2)}, \ldots, \mathbf{C}^{(N)} \rrbracket .
\end{aligned}
$$

The higher-order singular value decomposition (HOSVD) is a special case of the Tucker decomposition, where the core tensor is all-orthogonal [25], and the factor matrices are the unitary left singular matrices of the mode- $n$ unfolding of $\mathcal{A}$.

\section{SySTEM MODEL}

As shown in Fig. 11 a BS is equipped with an $M_{\mathrm{bs}}$-antenna large-scale hybrid mmWave UCyA consisting of $M_{\mathrm{v}}$ vertically placed uniform circular arrays (UCAs). Each of the UCAs is on a horizontal plane with $M_{\mathrm{h}}$ elements, and $M_{\mathrm{bs}}=M_{\mathrm{v}} M_{\mathrm{h}}$. Let $r$ be the radius of the $\mathrm{UCyA}$, and $h$ be the vertical spacing between any two adjacent vertical elements. We assume that there are $K$ IoT devices, each equipped with a single antenna 1 . Each device has a dominating path and different devices have separable and resolvable paths. Hence, $K$ signal paths are received by the $\mathrm{BS}$. The received signal sample at the $m_{\mathrm{t}}$-th time frame $\left(m_{\mathrm{t}}=1, \ldots, M_{\mathrm{t}}\right)$ can be expressed as [26]

$$
\mathbf{x}_{m_{\mathrm{t}}}=\sum_{k=1}^{K} s_{m_{\mathrm{t}}, k} \mathbf{B}^{H} \mathbf{a}_{\mathrm{bs}}\left(\phi_{k}, \theta_{k}\right)+\mathbf{n}_{m_{\mathrm{t}}}
$$

where $\phi_{k}$ and $\theta_{k}$ are the azimuth and elevation DoAs of the $k$-th device, respectively; $\mathbf{a}_{\mathrm{bs}}\left(\phi_{k}, \theta_{k}\right) \in \mathbb{C}^{M_{\mathrm{bs}} \times 1}$ denotes the steering vector of the hybrid $\mathrm{UCyA} ; s_{m_{\mathrm{t}}, k}$ is the received symbol of the $k$-th device at the $m_{\mathrm{t}}$-th time frame; $\mathbf{n}_{m_{\mathrm{t}}} \in$ $\mathbb{C}^{M_{\mathrm{bs}} \times 1}$ denotes the additive white Gaussian noise (AWGN); B $\in \mathbb{C}^{M_{\mathrm{bs}} \times M_{\mathrm{bsd}}}$ is the hybrid beamforming matrix; and $M_{\mathrm{bsd}}$ is the number of data streams.

\footnotetext{
${ }^{1}$ The proposed technique can be readily applied when multiple antennas are deployed at a device. In that case, the paths originating from different antennas can be distinguished by transmitting different pilot signals.
}

Given the structure of the UCyA, the array steering vector $\mathbf{a}_{\mathrm{bs}}\left(\phi_{k}, \theta_{k}\right)$ can be written as $\mathbf{a}_{\mathrm{bs}}\left(\phi_{k}, \theta_{k}\right)=\mathbf{a}_{\mathrm{v}}\left(\theta_{k}\right) \otimes$ $\mathbf{a}_{\mathbf{h}}\left(\theta_{k}, \phi_{k}\right)$, where $\mathbf{a}_{\mathbf{v}}\left(\theta_{k}\right)$ and $\mathbf{a}_{\mathbf{h}}\left(\theta_{k}, \phi_{k}\right)$ are the vertical and horizontal array steering vectors with their elements given by

$$
\begin{gathered}
{\left[\mathbf{a}_{\mathrm{v}}\left(\theta_{k}\right)\right]_{m_{\mathrm{v}}}=a_{\mathrm{v}, m_{\mathrm{v}}}\left(\theta_{k}\right)} \\
=\frac{1}{\sqrt{M_{\mathrm{v}}}} \exp \left(-j \frac{2 \pi}{\lambda} h\left(m_{\mathrm{v}}-1\right) \cos \left(\theta_{k}\right)\right), \\
{\left[\mathbf{a}_{\mathrm{h}}\left(\theta_{k}, \phi_{k}\right)\right]_{m_{\mathrm{h}}}=a_{\mathrm{h}, m_{\mathrm{h}}}\left(\theta_{k}, \phi_{k}\right)} \\
=\frac{1}{\sqrt{M_{\mathrm{h}}}} \exp \left(j \frac{2 \pi}{\lambda} r \sin \left(\theta_{k}\right) \cos \left(\phi_{k}-\varphi_{m_{\mathrm{h}}}\right)\right),
\end{gathered}
$$

where $\lambda$ is the wavelength, $m_{\mathrm{v}}=1, \ldots, M_{\mathrm{v}}$ and $m_{\mathrm{h}}=$ $1, \ldots, M_{\mathrm{h}} \cdot \varphi_{m_{\mathrm{h}}}=2 \pi\left(m_{\mathrm{h}}-1\right) / M_{\mathrm{h}}$ is the difference of the central angles between the $m_{\mathrm{h}}$-th antenna and the first antenna of each UCA. The geometric model of the UCyA is shown in Fig. 11 In this paper, the antenna array can be reasonably treated as a phased array because the signal bandwidth $B$ is much smaller than the carrier frequency $f$, i.e. $B \ll f$, and the signals are narrowband.

\section{Proposed Nested 3D Hybrid UCyA}

In this section, we design the hybrid beamformer $\mathbf{B}$ for performing channel estimation. $\mathbf{B}=\mathbf{B}_{\mathrm{rf}} \mathbf{B}_{\mathrm{bb}} \in \mathbb{C}^{M_{\mathrm{bs}} \times M_{\mathrm{bsd}}}$ can be decoupled between an analog beamforming matrix $\mathbf{B}_{\mathrm{rf}} \in$ $\mathbb{C}^{M_{\mathrm{bs}} \times M_{\mathrm{rf}}}$ and a digital beamforming matrix $\mathbf{B}_{\mathrm{bb}} \in \mathbb{C}^{M_{\mathrm{rf}} \times M_{\mathrm{bsd}}}$. Here, $M_{\mathrm{rf}}$ is the number of RF chains. We first briefly review the concept of difference coarray and sparse array, which are heavily used in this paper. Then, we introduce the $\mathbf{B}$ design process in detail.

\section{A. Review of Sparse Arrays}

Definition 1 (Difference Coarray): For an antenna array with $N$ elements, $\mathbf{w}_{n}$ is the position of its $n$-th element, $n=1,2, \ldots, N$. Let $\mathbf{w}_{n} \in \mathbb{C}^{3 \times 1}$ denote the $3 \mathrm{D}$ coordinate of the $n$-th antenna array element. The locations of all array elements are collected in the set $\mathbb{D}_{\mathrm{a}}$, i.e., $\mathbb{D}_{\mathrm{a}}=\left\{\mathbf{w}_{n}\right\}$. The difference coarray of the antenna array is an (virtual) array with element positions given by the set $\mathbb{D}_{\mathrm{dc}}$ :

$$
\mathbb{D}_{\mathrm{dc}}=\left\{\mathbf{w}_{n_{1}}-\mathbf{w}_{n_{2}}\right\}, \forall n_{1}, n_{2}=1,2, \ldots, N .
$$

According to (10), the element positions of the difference coarray are the (self) differences between the locations of original physical antenna elements.

Based on the definition of difference coarray, we can define a cross difference coarray, which corresponds to the cross differences between the element locations of two arrays with $N$ and $M$ elements:

$$
\mathbb{D}_{\mathrm{cdc}}= \pm\left\{\mathbf{w}_{n}-\mathbf{w}_{m}\right\}, \forall n=1,2, \ldots, N, m=1,2, \ldots, M .
$$

According to (10) and 11), we can see that the concept of difference coarray arises naturally in the second-order statistics

\footnotetext{
${ }^{2}$ Much smaller is defined by $|(f \pm B) / f| \approx 1$. When $f=60 \mathrm{GHz}$ and
} $B \leq 2 \mathrm{GHz}$, it has $|(f \pm B) / f| \in[0.97,1.03]$. 


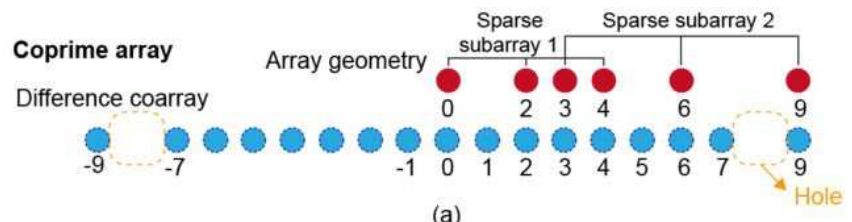

(a)

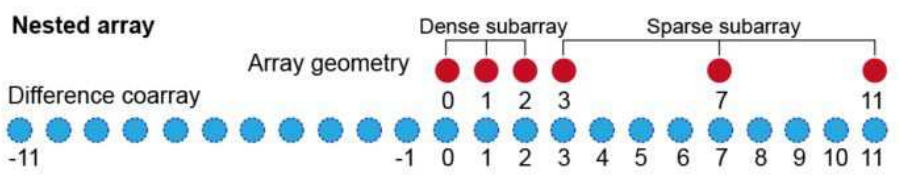

(b)

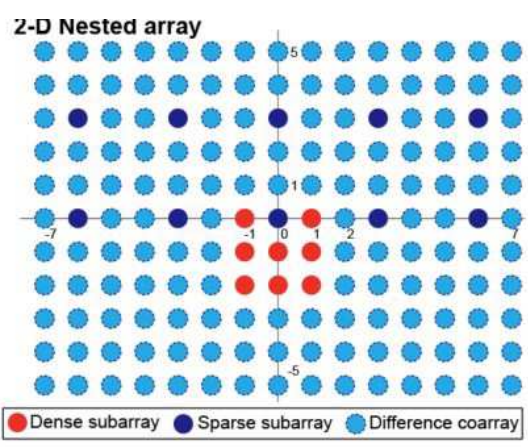

(c)

Fig. 2. An example of (a) coprime array, which is composed of two sparse subarrays: one with $N=3$ elements and separation $M=2$, and another one with $2 M-1$ elements and separation $N$; (b) nested array, which is composed of a dense subarray with $N_{1}=3$ elements and separation 1 , and a sparse subarray with $N_{2}=3$ elements and separation $N_{1}+1$; and (c) 2-D nested array, which is composed of a $3 \times 3$ dense subarray and a $5 \times 2$ sparse subarray.

of the impinging signals. For example, we consider that a signal $\mathbf{x}_{\mathbf{w}_{n}} \in \mathbb{C}^{N \times 1}$ is received at the $n$-th element of an antenna array. The cross-correlation between the signals received at the $n_{1}$-th and $n_{2}$-th elements of the array is given by

$$
\mathrm{E}\left\{\mathbf{x}_{\mathbf{w}_{n_{1}}} \mathbf{x}_{\mathbf{w}_{n_{2}}}^{H}\right\}=\mathbf{R}_{\left(\mathbf{w}_{n_{1}}-\mathbf{w}_{n_{2}}\right)} \in \mathbb{C}^{N \times N}, \mathbf{w}_{n_{1}}, \mathbf{w}_{n_{2}} \in \mathbb{D}_{\mathrm{a}}
$$

where $\mathbf{R}_{\left(\mathbf{w}_{n_{1}}-\mathbf{w}_{n_{2}}\right)}$ can be viewed as a signal sample received by a (larger) difference coarray with virtual array elements located at $\left(\mathbf{w}_{n_{1}}-\mathbf{w}_{n_{2}}\right) \in \mathbb{D}_{\mathrm{dc}}[12]$.

By adequately designing the element locations, i.e., $\mathbb{D}_{\mathrm{a}}$, we can increase the number of virtual elements in the difference coarray after computing the autocorrelation. If we use the samples from the difference coarray to perform spectral estimation, the parameters of much more targets can be estimated.

We proceed to introduce the concept of sparse array. An array is said to be sparse if the spacing between a majority or all of adjacent elements is more than one (halfwavelength) [12], [15]. By applying the concept of sparse array to antenna design, we can significantly improve the number of distinguishable targets using a small number of physical antenna elements [27]. Some well-known 1-D sparse arrays include MRA [13], MHA [14], nested arrays [12], and coprime arrays [15]. With $O(N)$ physical array elements, both MRA and MHA can construct difference coarrays with the size of $O\left(N^{2}\right)$. However, their geometries need to be constructed by using searching algorithms, e.g., integer programming [14], [28]. Nested and coprime arrays were proposed in [12], [15] with closed-form expressions for element locations, and both of them can construct difference coarrays with the same DoF as MRA and MHA. An example of nested and coprime arrays, and their difference coarrays are shown in Figs. 2(a) and 2(b). Nested arrays can offer larger difference coarray DoF than coprime arrays, as shown in Fig. 2(b), where both of them have six physical elements. In addition, the difference coarrays of nested arrays consist of evenly spaced virtual elements with no holes, so that the subspace-based estimation algorithms, such as MUSIC and ESPRIT, can be utilized on the coarray domain without creating ambiguities [28]. For the details of

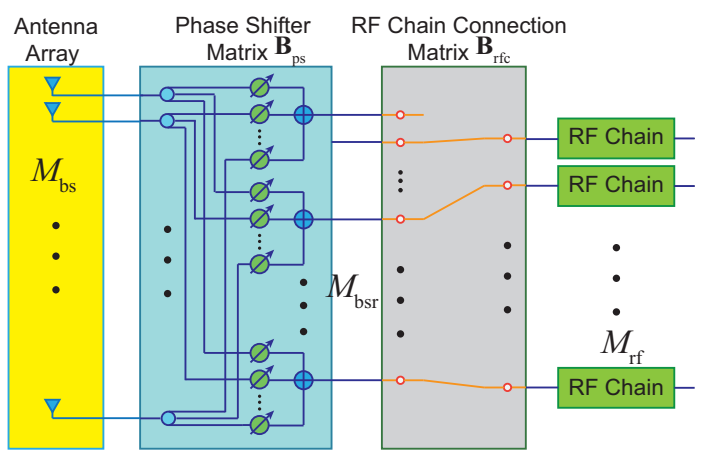

Fig. 3. The block diagram of RF front-end structure.

these arrays, interested readers can refer to [12] $-[15]$.

In the next subsection, we design the phase shifter matrix $\mathbf{B}_{\mathrm{ps}}$. $\mathbf{B}_{\mathrm{ps}}$ can transform the UCyA steering vectors from the element space into a phase space, where the phases of the array steering vectors are linear to the element locations. From (12), we see that if we want to construct a difference coarray with a similar geometry to that of the original array, e.g., the UCyA in our system, the phase of the array steering vectors should vary linearly with the element locations. However, due to the special geometry of the UCyA, if we directly calculate the cross correlation of the array steering vectors, it would generate a virtual non-UCyA composed of multiple non-UCAs [24], leading to an increased computational complexity and degraded the estimation accuracy.

\section{B. Phase-Space Transformation}

The analog beamforming matrix $\mathbf{B}_{\mathrm{rf}}=\mathbf{B}_{\mathrm{ps}} \mathbf{B}_{\mathrm{rfc}} \in \mathbb{C}^{M_{\mathrm{bs}} \times M_{\mathrm{rf}}}$ is composed of a phase shifter matrix $\mathbf{B}_{\mathrm{ps}} \in \mathbb{C}^{M_{\mathrm{bs}} \times M_{\mathrm{bsr}}}$ and an RF-chain connection matrix $\mathbf{B}_{\mathrm{rfc}} \in \mathbb{C}^{M_{\mathrm{bsr}} \times M_{\mathrm{rf}}}$, where $M_{\mathrm{bsr}}$ is the number of output ports of the phase-shifter matrix. An illustration of the RF front-end structure is shown in Fig. 3. Here, we design $\mathbf{B}_{\mathrm{ps}}$ based on circular phase-space transformation [29], to transform the nonlinear phase of UCyA steering vectors to be linear to the element locations.

We decouple $\mathbf{B}_{\mathrm{ps}}$ between the vertical and horizontal planes, i.e., $\mathbf{B}_{\mathrm{ps}}=\mathbf{B}_{\mathrm{vps}} \otimes \mathbf{B}_{\mathrm{hps}}$ with $\mathbf{B}_{\mathrm{vps}} \in \mathbb{C}^{M_{\mathrm{v}} \times M_{\mathrm{vr}}}$ and $\mathbf{B}_{\mathrm{hps}} \in$ 
$\mathbb{C}^{M_{\mathrm{h}} \times M_{\mathrm{hr}}}$, and thus $M_{\mathrm{bsr}}=M_{\mathrm{vr}} M_{\mathrm{hr}}$, where $M_{\mathrm{vr}}$ and $M_{\mathrm{hr}}$ are the number of the phase-shifter output ports along the vertical and horizontal directions, respectively. According to the phase-space transformation of UCAs [29], we design $\mathbf{B}_{\text {hps }}$ as $\left[\mathbf{B}_{\mathrm{hps}}\right]_{m_{\mathrm{h}}, m_{\mathrm{hr}}+P+1}=e^{-j \frac{2 \pi\left(m_{\mathrm{h}}-1\right)}{M_{\mathrm{h}}} m_{\mathrm{hr}}}$, where $M_{\mathrm{hr}}=2 P+1$, $m_{\mathrm{hr}}=-P,-P+1, \ldots, P$, and $P$ is the highest phase-space dimension. Thus, the $M_{\mathrm{h}}$-dimensional array steering vector $\mathbf{a}_{\mathbf{h}}\left(\theta_{k}, \phi_{k}\right)$ can be transformed into a $(2 P+1)$-dimensional phase space, i.e., $\mathbf{a}_{\mathrm{hps}}\left(\theta_{k}, \phi_{k}\right)=\mathbf{B}_{\mathrm{hps}}^{H} \mathbf{a}_{\mathrm{h}}\left(\theta_{k}, \phi_{k}\right) \in \mathbb{C}^{(2 P+1) \times 1}$. The value of the highest phase-space dimension, $P$, can be configured based on the following theorem.

Theorem 1. Suppose that $M_{h} \geq\lfloor 4 \pi r / \lambda\rfloor$. If the highest phase-space dimension, $P$, is larger than $\lfloor 2 \pi r / \lambda\rfloor$ and smaller than $M_{h r} / 2$, then the elements in the phase-space response can be approximated by

$$
a_{\text {hps }, p}\left(\theta_{k}, \phi_{k}\right) \approx \sqrt{M_{h}} j^{p} J_{p}\left(\gamma\left(\theta_{k}\right)\right) \exp \left(-j p \phi_{k}\right),
$$

where $\gamma\left(\theta_{k}\right)=2 \pi r \sin \left(\theta_{k}\right) / \lambda, p=-P,-P+1, \ldots, P$, and $J_{p}\left(\gamma\left(\theta_{k}\right)\right)$ is the Bessel function of the first kind of order $p$.

Proof. See Appendix I.

We set $\mathbf{B}_{\mathrm{vps}}=\mathbf{I}_{M_{\mathrm{v}}}$ to preserve the recurrence relations among UCAs. According to Theorem 1, the array steering vectors $\mathbf{a}_{\mathrm{bs}}\left(\theta_{k}, \phi_{k}\right)$ after the hybrid beamformer is given by

$$
\begin{aligned}
& \mathbf{a}_{\mathrm{bd}}\left(\theta_{k}, \phi_{k}\right)=\mathbf{B}^{H} \mathbf{a}_{\mathrm{bs}}\left(\theta_{k}, \phi_{k}\right) \\
& =\left(\mathbf{B}_{\mathrm{rf}} \mathbf{B}_{\mathrm{bb}}\right)^{H} \mathbf{a}_{\mathrm{bs}}\left(\theta_{k}, \phi_{k}\right) \\
& =\left(\left(\mathbf{B}_{\mathrm{vps}} \otimes \mathbf{B}_{\mathrm{hps}}\right) \mathbf{B}_{\mathrm{rfc}} \mathbf{I}_{M_{\mathrm{bsr}}}\right)^{H} \mathbf{a}_{\mathrm{bs}}\left(\theta_{k}, \phi_{k}\right) \\
& =\mathbf{B}_{\mathrm{rfc}}^{H}\left(\mathbf{B}_{\mathrm{vps}} \otimes \mathbf{B}_{\mathrm{hps}}\right)^{H} \mathbf{a}_{\mathrm{bs}}\left(\theta_{k}, \phi_{k}\right),
\end{aligned}
$$

where $\mathbf{B}_{\mathrm{bb}}$ is a diagonal matrix used to guarantee the power constraint [30]. Without loss of generality, we set $\mathbf{B}_{\mathrm{bb}}=\mathbf{I}_{M_{\mathrm{bsr}}}$ in this paper. According to two properties of the Khatri-Rao product: $(\mathbf{A} \otimes \mathbf{B})^{H}=\mathbf{A}^{H} \otimes \mathbf{B}^{H}$ and $(\mathbf{A} \otimes \mathbf{B})(\mathbf{C} \otimes \mathbf{D})=$ $\mathbf{A C} \otimes \mathbf{B D}$ [31], (14] can be rewritten as

$$
\begin{aligned}
& \mathbf{a}_{\mathrm{bd}}\left(\theta_{k}, \phi_{k}\right) \\
& =\mathbf{B}_{\mathrm{rfc}}^{H}\left(\mathbf{B}_{\mathrm{vps}}^{H} \otimes \mathbf{B}_{\mathrm{hps}}^{H}\right)\left(\mathbf{a}_{\mathrm{v}}\left(\theta_{k}\right) \otimes \mathbf{a}_{\mathbf{h}}\left(\theta_{k}, \phi_{k}\right)\right) \\
& =\mathbf{B}_{\mathrm{rfc}}^{H}\left[\left(\mathbf{B}_{\mathrm{vps}}^{H} \mathbf{a}_{\mathrm{v}}\left(\theta_{k}\right)\right) \otimes\left(\mathbf{B}_{\mathrm{hps}}^{H} \mathbf{a}_{\mathrm{h}}\left(\theta_{k}, \phi_{k}\right)\right)\right] \\
& =\mathbf{B}_{\mathrm{rfc}}^{H}\left[\mathbf{a}_{\mathrm{vps}}\left(\theta_{k}\right) \otimes \mathbf{a}_{\mathrm{hps}}\left(\theta_{k}, \phi_{k}\right)\right],
\end{aligned}
$$

where $\mathbf{a}_{\mathrm{vps}}\left(\theta_{k}\right) \in \mathbb{C}^{M_{\mathrm{vr}} \times 1}, \mathbf{a}_{\mathrm{hps}}\left(\theta_{k}, \phi_{k}\right) \in \mathbb{C}^{M_{\mathrm{hr}} \times 1}, M_{\mathrm{vr}}=M_{\mathrm{v}}$, and $M_{\mathrm{hr}}=2 P+1$.

According to Theorem 1, we have

$$
\begin{gathered}
\mathbf{a}_{\mathrm{vps}}\left(\theta_{k}\right)=\mathbf{a}_{\mathrm{v}}\left(\theta_{k}\right)=\mathbf{I}_{M_{\mathrm{v}}} \mathbf{a}_{\mathrm{v}}\left(\theta_{k}\right), \\
a_{\mathrm{hps}, m_{\mathrm{hr}}}\left(\theta_{k}, \phi_{k}\right)=\left[\mathbf{B}_{\mathrm{hps}}^{H}\right]_{m_{\mathrm{hr}}+P+1,:} \mathbf{a}_{\mathrm{h}}\left(\theta_{k}, \phi_{k}\right) \\
\approx \sqrt{M_{\mathrm{h}}} j^{m_{\mathrm{hr}}} J_{m_{\mathrm{hr}}}\left(\gamma\left(\theta_{k}\right)\right) \exp \left(-j m_{\mathrm{hr}} \phi_{k}\right) .
\end{gathered}
$$

From (16) and (17), we see that, through the proposed $\mathbf{B}_{\mathrm{ps}}$, the phases of the array steering vectors become linear to the element locations. This is important to exploit the property of the sparse array theory to design the RF-chain connection matrix $\mathbf{B}_{\mathrm{rfc}}$.

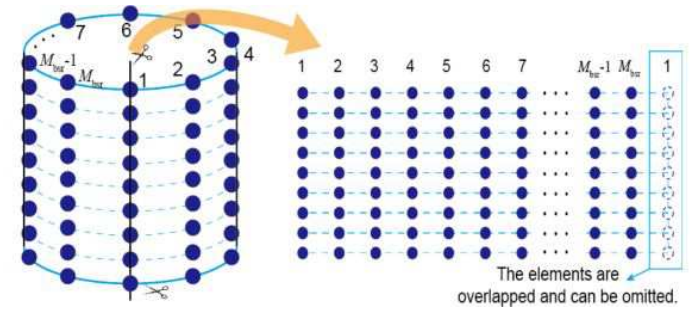

Fig. 4. An illustration of unfolding phase-shifter output ports of a 3-D UCyA to be a 2-D array.

\section{RF-Chain Connection Network Design}

In this subsection, we apply the sparse array technique to design $\mathbf{B}_{\mathrm{rfc}}$, which enables the DoAs of a large number of devices to be estimated with a marginal accuracy loss while significantly reducing the number of required RF chains. We aim to use as few RF chains as possible to achieve the same, or even larger, DoF than the fully connected beamforming array 3 . This objective is different from the previous sparse array researches, which have typically focused on maximizing the size of difference coarrays under the constraint of a fixed number of physical antenna elements.

We first flatten the 3-D RF-chain connection network of UCyA into a 2-D plane, as shown in Fig. 4, by disjoining the RF-chain connection network at the first column phase shifters of every UCA. Different from typical 2-D arrays, due to the periodicity of UCAs, the first and the last phase-shifter output ports of every row in the flattened 2-D RF-chain connection network are identical, as shown in Fig. 4 where the dotted circles denote the last-column phase shifters.

After the flattening processing, the 3-D RF-chain connection network becomes a quasi-2D rectangular array with size of $\left(M_{\mathrm{hr}}+1\right) \times M_{\mathrm{vr}}$, where the increased dimension is due to the repeated phase-shifter output ports, as shown in Fig. 4 The idea of 2-D sparse arrays can be applied to design a 3-D RFchain connection network of UCyA 4 . In this paper, we design the RF-chain connection network based on a 2-D nested array. This is because (1) a nested array can generate larger holefree difference coarrays than a coprime array under the same setting, as discussed in Section III-A; and (2) it has simple closed-form expressions for a large number of elements, which cannot be achieved in MRA and MHA. There are also some other frequently-used 2-D sparse arrays, e.g., hourglass arrays and open box arrays (OBAs) [28], [32]. We will compare the RF-chain connection networks designed based on those array geometries with our design in Section V-C.

Our proposed sparse RF-chain connection network is developed from the "Configuration II" nested array [17]. In the general "Configuration II" nested array, when there are

\footnotetext{
${ }^{3}$ Due to the use of phase shifter network, the antenna DoF of UCyA depends on the scale of the phase shifter network. Thus, if we use a fully connected beamforming array, $(2 P+1) M_{\mathrm{v}} \mathrm{RF}$ chains are needed, which can provide $\mathcal{O}\left(P M_{\mathrm{V}}\right)$ DoFs.

${ }^{4}$ Although the RF-chain connection network actually does not have the exact shape, according to the array steering vectors in [16 and [17, we can also regard the network as an UCyA.
} 
$N_{\text {dense }}=N_{\mathrm{vd}} N_{\text {hd }}-1$ and $N_{\text {sparse }}=N_{\text {vs }} N_{\text {hs }}$ elements in the dense and sparse subarrays, respectively, the constructed holefree difference coarray has $N_{\mathrm{dc}}=N_{\mathrm{vdc}} N_{\mathrm{hdc}}=\left(2 N_{\mathrm{vd}} N_{\mathrm{vs}}-\right.$ 1) $N_{\text {hd }} N_{\text {hs }}$ elements [17], as shown in Fig. 2(c). Here, $N_{\mathrm{vd}}$ and $N_{\text {hd }}$ are the numbers of elements in the dense subarray along the vertical and horizontal directions, respectively; $N_{\mathrm{vs}}$ and $N_{\text {hs }}$ are the numbers of elements in the sparse subarray along the vertical and horizontal directions, respectively; and $N_{\mathrm{vdc}}$ and $N_{\text {hdc }}$ are the numbers of elements in the difference coarray along the vertical and horizontal directions, respectively. We wish to find the distribution of the RF chains between the sparse and the dense arrays that use as few RF chains as possible to achieve the same DoF as the fully connected beamforming array.

Due to the above-mentioned periodicity of UCAs, when we apply the sparse array technique into our hybrid frontend design, two cases need to be considered for UCyAs. Fig. 5 (a) shows the first case, where the first and the last columns of the difference coarray overlap. From Fig. 5(a), we can see that because one column (first or last) of the difference coarray is redundant, two elements of the sparse subarray can be omitted to reduce the element number. A drawback is that this case requires $M_{\mathrm{hr}}=\left(N_{\mathrm{hs}}-1\right) N_{\mathrm{hd}}$, which would impose a strict requirement on the number of phase shifters on the horizontal plane. The second case is shown in Fig. 5 (b), which requires the constructed difference coarray to be larger than the original UCyA, to achieve the same DoF as a fully connected beamforming array on the horizontal space. In this case, $N_{\mathrm{hd}} N_{\mathrm{hs}} \geq M_{\mathrm{hr}}>\left(N_{\mathrm{hs}}-1\right) N_{\mathrm{hd}} / 2$.

In our system, due to the new phase shifter network designed in Section III-B, we have $M_{\mathrm{hr}}=2 P+1$ (which is an odd number). Since in the Configuration II nested array, the dense and sparse subarrays are symmetric, i.e., both $N_{\mathrm{hs}}$ and $N_{\text {hd }}$ are odd, we have $\left(N_{\text {hs }}-1\right) N_{\text {hd }}$ is even, and only the above-mentioned second case needs to be considered in our system. We formulate the optimization problem as

$$
\begin{aligned}
\min _{N_{\mathrm{vd}}, N_{\mathrm{hd}}, N_{\mathrm{vs}}, N_{\mathrm{hs}} \in \mathbb{Z}^{+}} & M_{\mathrm{rf}}=N_{\mathrm{vd}} N_{\mathrm{hd}}+N_{\mathrm{vs}} N_{\mathrm{hs}}-1 \\
\text { s.t. } & (\mathrm{C} 1): 2 N_{\mathrm{vd}} N_{\mathrm{vs}}-1 \geq M_{\mathrm{vr}}, \\
& \text { (C2): } N_{\mathrm{hd}} N_{\mathrm{hs}} \geq M_{\mathrm{hr}}, \\
& \text { (C3): } N_{\mathrm{vd}}, N_{\mathrm{hd}}>1, \\
& \text { (C4): } N_{\mathrm{hd}} \text { is odd and } N_{\mathrm{vd}} / N_{\mathrm{hd}} \in \mathbb{Z}^{+} .
\end{aligned}
$$

(C1) and (C2) guarantee the DoF requirements for the constructed difference coarray of the RF-chain connection network. (C3) avoids solutions that degenerate to 1-D arrays. (C4) is due to the fact that the dense array in Configuration II is symmetrical, and $N_{\mathrm{hd}}$ and $N_{\mathrm{vd}}$ are invariant factors [18] of the array distribution matrix.

The solution for (18) can be obtained by adopting the following strategy. According to (C1) and (C2), since $N_{\mathrm{vs}}, N_{\mathrm{hs}} \in$ $\mathbb{Z}^{+}$, we can obtain $N_{\mathrm{vs}}=\left\lceil\left(M_{\mathrm{vr}}-1\right) / 2 N_{\mathrm{vd}}\right\rceil$ and $N_{\mathrm{hs}}=$ $\left\lceil M_{\mathrm{hr}} / N_{\mathrm{hd}}\right\rceil$. The optimization problem (18) becomes

$$
\begin{gathered}
\min _{N_{\mathrm{vd}}, N_{\mathrm{hd}} \in \mathbb{Z}^{+}} M_{\mathrm{rf}}=N_{\mathrm{vd}} N_{\mathrm{hd}}+\left\lceil\frac{\left(M_{\mathrm{vr}}-1\right) / 2}{N_{\mathrm{vd}}}\right\rceil\left\lceil\frac{M_{\mathrm{hr}}}{N_{\mathrm{hd}}}\right\rceil \\
\text { s.t. (C3) and (C4) }
\end{gathered}
$$

Given $M_{\mathrm{vr}}$ and $M_{\mathrm{hr}}$, we see that (19) exhibits the form of $y=x+\frac{a}{x}$, where $a>0$ is a constant and $y=x+\frac{a}{x} \geq 2 \sqrt{a}$. Because $y=2 \sqrt{a}$ iff $x=$ $\frac{a}{x}$, the minimum $M_{\mathrm{rf}}$ can be obtained when the difference between $N_{\mathrm{vd}} N_{\mathrm{hd}}$ and $\left\lceil\left(M_{\mathrm{vr}}-1\right) / 2 N_{\mathrm{vd}}\right\rceil\left\lceil M_{\mathrm{hr}} / N_{\mathrm{hd}}\right\rceil$ is the smallest. Since $N_{\mathrm{vd}}, N_{\mathrm{hd}}, N_{\mathrm{vs}}, N_{\mathrm{hs}} \in \mathbb{Z}^{+}$, we can determine the approximate value ranges of $N_{\mathrm{vd}} N_{\text {hd }}$ and $\left\lceil\left(M_{\mathrm{vr}}-1\right) / 2 N_{\mathrm{vd}}\right\rceil\left\lceil M_{\mathrm{hr}} / N_{\mathrm{hd}}\right\rceil$, and (19) is an integer programming problem. According to (C3) and (C4), the optimal solutions of $N_{\mathrm{vd}}, N_{\mathrm{hd}}, N_{\mathrm{vs}}$, and $N_{\mathrm{hs}}$ to 19 ) can be obtained by using brute-force search with the value range between $N_{\mathrm{vd}} N_{\mathrm{hd}}$ and $\left\lceil\left(M_{\mathrm{vr}}-1\right) / 2 N_{\mathrm{vd}}\right\rceil\left\lceil M_{\mathrm{hr}} / N_{\mathrm{hd}}\right\rceil$.

In the proposed sparse RF-chain connection network, the RF chains only need to connect the phase shifters located in the dense and sparse subarrays. Based on the calculated values of $N_{\text {vd }}, N_{\text {hd }}, N_{\text {vs }}$, and $N_{\text {hs }}$, now we provide the element locations in the dense and sparse arrays. For illustration convenience, we define the overlapping point of the sparse and dense arrays as the origin of the nested array 5 . Let $\mathbf{m}_{\mathrm{sp}}=\left(m_{\mathrm{v}_{\mathrm{S}} \mathrm{sp}}, m_{\mathrm{h} \_\mathrm{sp}}\right)$ and $\mathbf{m}_{\mathrm{de}}=\left(m_{\mathrm{v} \_ \text {de }}, m_{\mathrm{h} \_ \text {de }}\right)$ as the locations of elements in the dense and sparse arrays (to which the RF chains connect), respectively. We have

$$
\begin{aligned}
& \left\{\begin{array}{l}
m_{\mathrm{v} \_ \text {sp }}=N_{\mathrm{vd}}\left(n_{\mathrm{vs}}-1\right), \\
m_{\mathrm{h} \_\mathrm{sp}}=N_{\mathrm{hd}}\left(-N_{\mathrm{hs}} / 2+n_{\mathrm{hs}}-1 / 2\right),
\end{array}\right. \\
& \left\{\begin{array}{l}
m_{\mathrm{v} \_\mathrm{de}}=-N_{\mathrm{vd}}+n_{\mathrm{vd}}, \\
m_{\mathrm{h} \_\mathrm{de}}=-\left(N_{\mathrm{hd}}-1\right) / 2+n_{\mathrm{hd}}-1,
\end{array}\right.
\end{aligned}
$$

where $n_{\mathrm{vs}}=1,2, \ldots, N_{\mathrm{vs}} ; n_{\mathrm{hs}}=1,2, \ldots, N_{\mathrm{hs}} ; n_{\mathrm{vd}}=$ $1,2, \ldots, N_{\mathrm{vd}} ;$ and $n_{\mathrm{hd}}=1,2, \ldots, N_{\mathrm{hd}}$. Let $\mathbb{M}_{\mathrm{rf} \_\mathrm{d}}=$ $\left\{\mathbf{m}_{\mathrm{v}_{-} \text {de }} \otimes \mathbf{m}_{\mathrm{h}_{-} \text {de }}\right\}$ and $\mathbb{M}_{\mathrm{rf}_{\mathrm{f}} \mathrm{s}}=\left\{\mathbf{m}_{\mathrm{v}_{-} \text {sp }} \otimes \mathbf{m}_{\mathrm{h}_{\text {_sp }}}\right\}$ denote the sets of the RF-chain connection points in the dense and sparse arrays, respectively. $\mathbf{m}_{\text {v_de }} \in \mathbb{C}^{N_{\text {vd }} \times 1}$ and $\mathbf{m}_{\mathrm{h}_{\text {_de }}} \in \mathbb{C}^{N_{\text {hd }} \times 1}$ are the element locations of the dense array along the vertical and horizontal directions, respectively. $\mathbf{m}_{\mathrm{v}-\mathrm{sp}} \in \mathbb{C}^{N_{\mathrm{vs}} \times 1}$ and $\mathbf{m}_{\mathrm{h} \_ \text {sp }} \in \mathbb{C}^{N_{\text {hs }} \times 1}$ are the element locations of the sparse array along the vertical and horizontal directions, respectively. The set of RF-chain connection points is $\mathbb{M}_{\mathrm{rfc}}=\left\{\mathbb{M}_{\mathrm{rf}_{-} \mathrm{d}} \cup \mathbb{M}_{\mathrm{r}_{\mathrm{r}}}\right\}$. The constructed RF-chain connection matrix $\mathbf{B}_{\mathrm{rfc}}$ is given by

$$
\begin{aligned}
& {\left[\mathbf{B}_{\mathrm{rfc}}\right]_{m_{\mathrm{bsr}}, m_{\mathrm{rf}}}=} \\
& \begin{cases}1, & \text { if } m_{\mathrm{bsr}} \in \mathbb{M}_{\mathrm{rfc}} \text { and } \\
& {\left[\mathbf{B}_{\mathrm{rfc}}\right]_{m_{\mathrm{bsr}}^{\prime} \neq m_{\mathrm{bsr}}, m_{\mathrm{rf}}}=\left[\mathbf{B}_{\mathrm{rfc}}\right]_{m_{\mathrm{bsr}}, m_{\mathrm{rff}}^{\prime} \neq m_{\mathrm{rf}}}=0 ;} \\
0, & \text { otherwise. }\end{cases}
\end{aligned}
$$

By deploying the proposed sparse RF-chain connection network and using the second-order statistics of the received

\footnotetext{
${ }^{5}$ Because the parameter estimation depends on the difference between array elements, the changed absolute positions of array elements does not effect the estimation performance.
} 


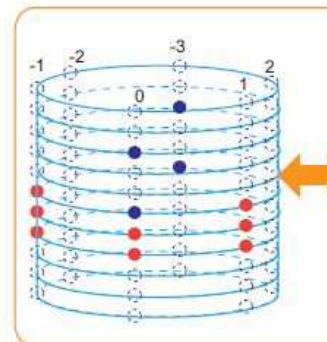

(a)

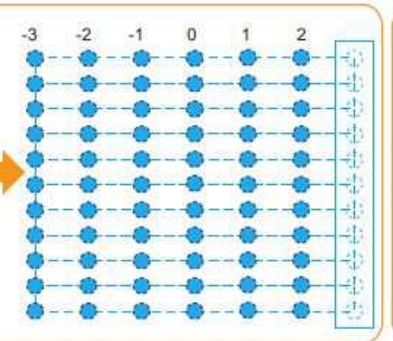

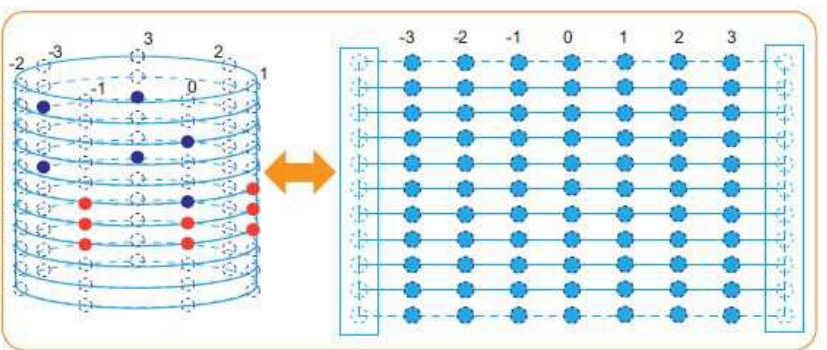

(b)

Fig. 5. Two cases of 3-D UCyA unfolding. The locations of the cylindrical post-phase-shifting ports in sparse and dense subarrays are highlighted with red and navy blue dots.

signal for channel estimation, the DoF of the proposed network is $O\left(\left(2 N_{\mathrm{vd}} N_{\mathrm{vs}}-1\right) \times M_{\mathrm{hr}}\right)$. In other words, according to [18], we can estimate the channel parameters of $\left(N_{\mathrm{vdc}}-1\right)\left(N_{\mathrm{hdc}}-1\right)$ devices by using the proposed hybrid front-end. Because there is an overlapping point at the origin, the total number of RF chains required in our network is $M_{\mathrm{rf}}=N_{\mathrm{vd}} N_{\mathrm{hd}}+N_{\mathrm{vs}} N_{\mathrm{hs}}-1$, as shown in 18 6 . Due to the periodicity of UCAs, there are only up to $N_{\mathrm{hdc}}=M_{\mathrm{hr}}$ virtual elements on the horizontal plane of the constructed 3-D difference coarray of the RFchain connection network. Along the vertical direction, the number of virtual elements is $N_{\mathrm{vdc}}=2 N_{\mathrm{vd}} N_{\mathrm{vs}}-1$.

According to the element locations of the dense and sparse arrays in (20) and (21), we also provide the element locations of the constructed difference coarray. Let $\mathbf{m}_{\mathrm{dc}}=$ $\left(m_{\mathrm{v}_{-} \mathrm{dc}}, m_{\mathrm{h}_{-} \mathrm{dc}}\right)$, where $m_{\mathrm{v}_{-} \mathrm{dc}}$ and $m_{\mathrm{h}_{\mathrm{d}} \mathrm{dc}}$ correspond to the locations along the vertical and horizontal directions, respectively. We have

$$
m_{\mathrm{v} \_\mathrm{dc}}=-\left(N_{\mathrm{vdc}}-1\right) / 2+n_{\mathrm{vdc}}-1=-N_{\mathrm{vd}} N_{\mathrm{vs}}+n_{\mathrm{vdc}},
$$

$$
m_{\mathrm{h} \_\mathrm{dc}}=-\left(N_{\mathrm{hdc}}-1\right) / 2+n_{\mathrm{hdc}}-1=-P+n_{\mathrm{hdc}}-1 \text {, }
$$

where $n_{\mathrm{vdc}}=1,2, \ldots, N_{\mathrm{vdc}}$ and $n_{\mathrm{hdc}}=1,2, \ldots, N_{\mathrm{hdc}}$. The shape of the constructed 3-D difference coarray RF-chain connection network is the same as the UCyA, but the former has a larger DoF.

The signals through the proposed RF-chain connection network are given by

$$
\mathbf{x}_{\mathrm{sn}, m_{\mathrm{t}}}=\sum_{k=1}^{K} s_{m_{\mathrm{t}}, k} \mathbf{a}_{\mathrm{sn}}\left(\phi_{k}, \theta_{k}\right)+\mathbf{n}_{\mathrm{sn}, m_{\mathrm{t}}},
$$

where

$$
\begin{aligned}
& \mathbf{a}_{\mathrm{sn}}\left(\phi_{k}, \theta_{k}\right) \\
& =\left[\begin{array}{c}
\mathbf{a}_{\mathrm{sn}, \mathrm{s}}\left(\phi_{k}, \theta_{k}\right) \\
\mathbf{a}_{\mathrm{sn}, \mathrm{d}}\left(\phi_{k}, \theta_{k}\right)
\end{array}\right]=\left[\begin{array}{c}
\mathbf{a}_{\mathrm{sn}, \mathrm{sv}}\left(\theta_{k}\right) \otimes \mathbf{a}_{\mathrm{sn}, \mathrm{sh}}\left(\theta_{k}, \phi_{k}\right) \\
\mathbf{a}_{\mathrm{sn}, \mathrm{dv}}\left(\theta_{k}\right) \otimes \mathbf{a}_{\mathrm{sn}, \mathrm{dh}}\left(\theta_{k}, \phi_{k}\right)
\end{array}\right] .
\end{aligned}
$$

The elements of $\mathbf{a}_{\mathrm{sn}, \mathrm{sv}}\left(\theta_{k}\right) \in \mathbb{C}^{N_{\mathrm{vs}} \times 1}$ and $\mathbf{a}_{\mathrm{sn}, \mathrm{sh}}\left(\theta_{k}, \phi_{k}\right) \in$ $\mathbb{C}^{N_{\mathrm{hs}} \times 1}$ are $a_{\mathrm{sn}, \mathrm{sv}, n_{\mathrm{vs}}}\left(\theta_{k}\right)=a_{\mathrm{vs}, m_{\mathrm{v} s \mathrm{p}}}\left(\theta_{k}\right)$ and $a_{\mathrm{sn}, \mathrm{sh}, n_{\mathrm{hs}}}\left(\theta_{k}, \phi_{k}\right)=$

\footnotetext{
${ }^{6}$ It can also be proved that the phase shifter at this location is useless and does not need to be connected [17]. However, for ease of description, here we assume that this phase shifter is connected in our network, which does not affect the results in this paper.
}

$a_{\mathrm{hs}, m_{\mathrm{h}-\mathrm{p} p}}\left(\theta_{k}, \phi_{k}\right), \quad$ respectively, where $\quad a_{\mathrm{vs}, m_{\mathrm{v}-\mathrm{sp}}}\left(\theta_{k}\right) \quad$ and $a_{\mathrm{hs}, m_{\mathrm{h}-\mathrm{sp}}}\left(\theta_{k}, \phi_{k}\right)$ are the array steering vectors of the sparse subarray along the vertical and horizontal directions, respectively. The elements of the array steering vectors of the dense subarray, i.e., $\mathbf{a}_{\mathrm{sn}, \mathrm{dv}}\left(\theta_{k}\right) \in \mathbb{C}^{N_{\mathrm{vd}} \times 1}$ and $\mathbf{a}_{\mathrm{sn}, \mathrm{dh}}\left(\theta_{k}, \phi_{k}\right) \in \mathbb{C}^{N_{\mathrm{hd}} \times 1}$, can be written in the same way. Here, $\mathbf{n}_{\mathrm{sn}, m_{\mathrm{t}}} \in \mathbb{C}^{M_{\mathrm{rf}} \times 1}$ is the noise component through the RF-chain connection network.

The signal model (25) can also be rewritten as

$$
\mathbf{x}_{\mathrm{sn}, m_{\mathrm{t}}}=\mathbf{A}_{\mathrm{sn}} \mathbf{s}_{m_{\mathrm{t}}}+\mathbf{n}_{\mathrm{sn}, m_{\mathrm{t}}}
$$

where $\mathbf{A}_{\mathrm{sn}}=\left[\mathbf{a}_{\mathrm{sn}}\left(\phi_{1}, \theta_{1}\right), \mathbf{a}_{\mathrm{sn}}\left(\phi_{2}, \theta_{2}\right), \ldots, \mathbf{a}_{\mathrm{sn}}\left(\phi_{K}, \theta_{K}\right)\right] \in$ $\mathbb{C}^{M_{\mathrm{rf}} \times K}$ and $\mathbf{s}_{m_{\mathrm{t}}}=\left[s_{m_{\mathrm{t}}, 1}, s_{m_{\mathrm{t}}, 2}, \ldots, s_{m_{\mathrm{t}}, K}\right]^{T} \in \mathbb{C}^{K \times 1}$.

By calculating the autocorrelation of $\mathbf{x}_{\mathrm{sn}, m_{\mathrm{t}}}$, we have

$$
\mathbf{R}_{\mathrm{sn}, m_{\mathrm{t}}}=\mathrm{E}\left\{\mathbf{x}_{\mathrm{sn}, m_{\mathrm{t}}} \mathbf{x}_{\mathrm{sn}, m_{\mathrm{t}}}^{H}\right\}=\mathbf{A}_{\mathrm{sn}} \mathbf{R}_{\mathrm{ss}, m_{\mathrm{t}}} \mathbf{A}_{\mathrm{sn}}^{H}+\mathbf{R}_{\mathrm{nn}, m_{\mathrm{t}}},
$$

where $\mathbf{R}_{\mathrm{ss}, m_{\mathrm{t}}}=\operatorname{diag}\left(\sigma_{\mathrm{s}, m_{\mathrm{t}}, 1}^{2}, \ldots, \sigma_{\mathrm{s}, m_{\mathrm{t}}, K}^{2}\right)$ and $\mathbf{R}_{\mathrm{nn}, m_{\mathrm{t}}}=$ $\operatorname{diag}\left(\sigma_{\mathrm{n}, m_{\mathrm{t}}, 1}^{2}, \ldots, \sigma_{\mathrm{n}, m_{\mathrm{t}}, K}^{2}\right)$ are the autocorrelation matrices of $\mathbf{s}_{m_{\mathrm{t}}}$ and $\mathbf{n}_{\mathrm{sn}, m_{\mathrm{t}}}$, respectively.

We vectorize $\mathbf{R}_{\mathrm{sn}, m_{\mathrm{t}}}$ as

$$
\mathbf{y}_{\mathrm{vR}, m_{\mathrm{t}}}=\operatorname{vec}\left(\mathbf{R}_{\mathrm{sn}, m_{\mathrm{t}}}\right)=\left[\mathbf{A}_{\mathrm{sn}}^{*} \diamond \mathbf{A}_{\mathrm{sn}}\right] \mathbf{d}_{m_{\mathrm{t}}}+\operatorname{vec}\left(\mathbf{R}_{\mathrm{nn}, m_{\mathrm{t}}}\right)
$$

where $\left[\mathbf{d}_{m_{\mathrm{t}}}\right]_{k, 1}=\sigma_{\mathrm{s}, m_{\mathrm{t}}, k}^{2}$, and $\sigma_{\mathrm{s}, m_{\mathrm{t}}, k}^{2}$ is the power of the $k$-th signal. The $k$-th column of the matrix $\left[\mathbf{A}_{\mathrm{sn}}^{*} \diamond \mathbf{A}_{\mathrm{sn}}\right]$ contains elements representing the cross-differences between sparse and dense subarrays, i.e., $a_{\mathrm{sn}, \mathrm{s}, \mathbf{m}_{\mathrm{sp}}}^{*}\left(\phi_{k}, \theta_{k}\right) a_{\mathrm{sn}, \mathrm{d}, \mathbf{m}_{\mathrm{de}}}\left(\phi_{k}, \theta_{k}\right)$ and $\quad a_{\mathrm{sn}, \mathrm{s}, \mathbf{m}_{\mathrm{de}}}^{*}\left(\phi_{k}, \theta_{k}\right) a_{\mathrm{sn}, \mathrm{d}, \mathbf{m}_{\mathrm{sp}}}\left(\phi_{k}, \theta_{k}\right), \quad$ and self-differences of sparse and dense subarrays, $\quad$ i.e., $\quad a_{\mathrm{sn}, \mathrm{s}, \mathbf{m}_{\mathrm{sp}, 1}}^{*}\left(\phi_{k}, \theta_{k}\right) a_{\mathrm{sn}, \mathrm{s}, \mathbf{m}_{\mathrm{sp}, 2}}\left(\phi_{k}, \theta_{k}\right) \quad$ and $a_{\mathrm{sn}, \mathrm{d}, \mathbf{m}_{\mathrm{de}, 1}}^{*}\left(\phi_{k}, \theta_{k}\right) a_{\mathrm{sn}, \mathrm{d}, \mathbf{m}_{\mathrm{de}, 2}}\left(\phi_{k}, \theta_{k}\right)$. Here, $\mathbf{m}_{\mathrm{sp}, 1}$ and $\mathbf{m}_{\mathrm{sp}, 2}$ denote that $a_{\mathrm{sn}, \mathrm{s}, \mathbf{m}_{\mathrm{sp}, 1}}\left(\phi_{k}, \theta_{k}\right)$ and $a_{\mathrm{sn}, \mathrm{s}, \mathbf{m}_{\mathrm{sp}, 2}}\left(\phi_{k}, \theta_{k}\right)$ are different elements in the sparse subarray, and $\mathbf{m}_{\mathrm{de}, 1}$ and $\mathbf{m}_{\mathrm{de}, 2}$ denote that $a_{\mathrm{sn}, \mathrm{d}, \mathbf{m}_{\mathrm{de}, 1}}\left(\phi_{k}, \theta_{k}\right)$ and $a_{\mathrm{sn}, \mathrm{d}, \mathbf{m}_{\mathrm{de}, 2}}\left(\phi_{k}, \theta_{k}\right)$ are different elements in the dense subarray.

We sort the rows of $\mathbf{y}_{\mathrm{vR}, m_{\mathrm{t}}}$ in the ascending order of their phases, and then remove the redundant rows with the same phases. Then, we can obtain the array steering vector of the difference coarray $\mathbf{A}_{\mathrm{df}} \in \mathbb{C}^{N_{\text {vdc }} N_{\text {hdc }} \times 1}$ from $\left[\mathbf{A}_{\mathrm{sn}}^{*} \diamond \mathbf{A}_{\mathrm{sn}}\right]$. We 
also calculate and store the mean of the "nonzero" rows of $\operatorname{vec}\left(\mathbf{R}_{\mathrm{nn}, m_{\mathrm{t}}}\right)$, and obtain

$$
\mathbf{y}_{\mathrm{df}, m_{\mathrm{t}}}=\mathbf{A}_{\mathrm{df}} \mathbf{d}_{m_{\mathrm{t}}}+\sigma_{\mathrm{n}}^{2} \mathbf{e}_{\mathrm{df}},
$$

where $\left[\mathbf{A}_{\mathrm{df}}\right]_{:, k}=\mathbf{a}_{\mathrm{df}}\left(\phi_{k}, \theta_{k}\right)=\mathbf{a}_{\mathrm{dfv}}\left(\theta_{k}\right) \otimes \mathbf{a}_{\mathrm{dfh}}\left(\theta_{k}, \phi_{k}\right)$ and $\mathbf{e}_{\mathrm{df}} \in \mathbb{C}^{N_{\text {vdc }} N_{\text {hdc }} \times 1}$ is a vector of all zeros except a " 1 " at the $(P+1) N_{\mathrm{vd}} N_{\mathrm{vs}}$-th entry. The element phases of $\mathbf{a}_{\mathrm{dfv}}\left(\theta_{k}\right) \in$ $\mathbb{C}^{N_{\text {vdc }} \times 1}$ and $\mathbf{a}_{\mathrm{dfh}}\left(\theta_{k}, \phi_{k}\right) \in \mathbb{C}^{N_{\mathrm{hdc}} \times 1}$ are given by

$$
\begin{gathered}
a_{\mathrm{dfv}, n_{\mathrm{vdc}}}\left(\theta_{k}\right)=\frac{1}{M_{\mathrm{v}}} \exp \left(-j \frac{2 \pi}{\lambda} h m_{\mathrm{v}_{-} \mathrm{dc}} \cos \left(\theta_{k}\right)\right), \\
a_{\mathrm{dfh}, n_{\mathrm{hdc}}}\left(\theta_{k}, \phi_{k}\right)=\xi_{m_{\mathrm{h}_{\mathrm{ddc}}}}\left(\theta_{k}\right) \exp \left(-j m_{\mathrm{h} \_\mathrm{dc}} \phi_{k}\right),
\end{gathered}
$$

where $\xi_{m_{\mathrm{h}_{\mathrm{d}} \mathrm{dc}}}\left(\theta_{k}\right)=M_{\mathrm{h}} j^{m_{\mathrm{h} \_\mathrm{dc}}} J_{m_{\mathrm{h}_{\mathrm{d}}, 1}}\left(\gamma\left(\theta_{k}\right)\right) J_{m_{\mathrm{h}_{-} \mathrm{ds}, 2}}\left(\gamma\left(\theta_{k}\right)\right)$, and $m_{\mathrm{h} \_\mathrm{dc}}=m_{\mathrm{h} \_\mathrm{ds}, 1}-m_{\mathrm{h} \_\mathrm{ds}, 2}\left(m_{\mathrm{h} \_\mathrm{ds}, 1}, m_{\mathrm{h} \_\mathrm{ds}, 2} \in \mathcal{M}_{\mathrm{h} \_\mathrm{ds}}\right)$. $\mathcal{M}_{\mathrm{h} \_\mathrm{ds}}=\left\{m_{\mathrm{h} \_\mathrm{de}}, m_{\mathrm{h} \_\mathrm{sp}}\right\}$ collects the horizontal locations of the elements in the dense and sparse arrays. (29) can be viewed as the signal $\mathbf{d}_{m_{\mathrm{t}}}$ received at an array with steering matrix $\mathbf{A}_{\mathrm{df}}$.

Now we formulate the received samples in the tensor form. We first decompose $\mathbf{y}_{\mathrm{df}, m_{\mathrm{t}}}$ into the vertical and horizontal domains (corresponding to the first and second modes of the tensor model), as given by $\mathbf{Y}_{\mathrm{df}, m_{\mathrm{t}}}=\operatorname{invec}\left(\mathbf{y}_{\mathrm{df}, m_{\mathrm{t}}}\right) \in$ $\mathbb{C}^{\left(2 N_{\mathrm{vd}} N_{\mathrm{vs}}-1\right) \times M_{\mathrm{hr}}}$. Then, we collect $\mathbf{Y}_{\mathrm{df}, m_{\mathrm{t}}}$ at all time frames, and store them in the time domain (corresponding to the third mode of the tensor model). Thus, the received samples can be expressed as

$$
\begin{aligned}
\mathcal{Y}_{\mathrm{df}} & =\left[\mathbf{Y}_{\mathrm{df}, 1} \sqcup_{3} \mathbf{Y}_{\mathrm{df}, 2} \sqcup_{3} \ldots \sqcup_{3} \mathbf{Y}_{\mathrm{df}, M_{\mathrm{t}}}\right] \\
& =\mathcal{A}_{\mathrm{df}} \times{ }_{3} \mathbf{D}+\mathcal{N}_{\mathrm{df}} \in \mathbb{C}^{N_{\mathrm{vdc}} \times N_{\mathrm{hdc}} \times M_{\mathrm{t}}},
\end{aligned}
$$

where $\mathbf{D}=\left[\mathbf{d}_{1}, \mathbf{d}_{2}, \ldots, \mathbf{d}_{M_{\mathrm{t}}}\right]^{T} \in \mathbb{C}^{M_{\mathrm{t}} \times K}, \mathcal{A}_{\mathrm{df}} \in$ $\mathbb{C}^{N_{\text {vdc }} \times N_{\text {hdc }} \times K}$ is known as the space-time response tensor [33], and $\mathcal{N}_{\text {df }}$ is the noise tensor model. Due to the above-mentioned process (29)-32), the elements of $\mathcal{N}_{\mathrm{df}}$ are all zeros except $\sigma_{\mathrm{n}}^{2}$ at $\left(0,0, m_{\mathrm{t}}\right), m_{\mathrm{t}}=1,2, \ldots, M_{\mathrm{t}}$. In (32), $\mathcal{A}_{\mathrm{df}}$ is obtained as

$$
\begin{gathered}
\mathcal{A}_{\mathrm{df}}=\left[\mathbf{a}_{\mathrm{dfv}}\left(\theta_{1}\right) \circ \mathbf{a}_{\mathrm{dfh}}\left(\theta_{1}, \phi_{1}\right) \sqcup_{3} \mathbf{a}_{\mathrm{dfv}}\left(\theta_{2}\right) \circ \mathbf{a}_{\mathrm{dfh}}\left(\theta_{2}, \phi_{2}\right)\right. \\
\left.\sqcup_{3} \ldots \sqcup_{3} \mathbf{a}_{\mathrm{dfv}}\left(\theta_{K}\right) \circ \mathbf{a}_{\mathrm{dfh}}\left(\theta_{K}, \phi_{K}\right)\right] .
\end{gathered}
$$

By substituting (33) into (32), we obtain

$$
\begin{aligned}
\mathcal{Y}_{\mathrm{df}} & =\sum_{k=1}^{K} \mathbf{a}_{\mathrm{dfv}}\left(\theta_{k}\right) \circ \mathbf{a}_{\mathrm{dfh}}\left(\theta_{k}, \phi_{k}\right) \circ[\mathbf{D}]_{:, k}+\mathcal{N}_{\mathrm{df}} \\
& =\llbracket \mathcal{Z}_{\mathrm{df}} ; \mathbf{A}_{\mathrm{dfv}}, \mathbf{A}_{\mathrm{dfh}}, \mathbf{D} \rrbracket+\mathcal{N}_{\mathrm{df}},
\end{aligned}
$$

where $\left[\mathbf{A}_{\mathrm{dfv}}\right]_{:, k}=\mathbf{a}_{\mathrm{dfv}}\left(\theta_{k}\right),\left[\mathbf{A}_{\mathrm{dfh}}\right]_{:, k}=\mathbf{a}_{\mathrm{dfh}}\left(\theta_{k}, \phi_{k}\right)$, and $\mathcal{Z}_{\mathrm{df}} \in$ $\mathbb{C}^{K \times K \times K}$ is an order-3 identity superdiagonal tensor 8 .

Eq. (32) shows that the elements of the equivalent signal matrix $\mathbf{D} \in \mathbb{C}^{M_{\mathrm{t}} \times K}$ are actually the received signal powers due to the autocorrelation calculation 27 . To build a full-rank matrix D for DoA estimation, one would need to assume that

\footnotetext{
${ }^{7}$ All the rows of $\operatorname{vec}\left(\mathbf{R}_{\mathrm{nn}, m_{\mathrm{t}}}\right)$ with nonzero value correspond to the phase difference of 0 in the different coarray, which are produced by the self difference of sparse and dense subarrays. Because the noise is temporally and spatially white with power $\sigma_{\mathrm{n}}^{2}$, by averaging the value of these rows, we have $\sigma_{\mathrm{n}}^{2}=\sum_{k=1}^{K} \sigma_{\mathrm{n}, m_{\mathrm{t}}, k}^{2}$.

${ }^{8} \mathrm{~A}$ tensor $\mathcal{A} \in \mathbb{C}^{I_{1} \times I_{2} \times \cdots \times I_{N}}$ is diagonal if $a_{i_{1} i_{2} \cdots i_{N}} \neq 0$ only if $i_{1}=$ $i_{2}=\cdots=i_{N}$. When $I_{1}=I_{2}=\cdots=I_{N}, \mathcal{A}$ is called as superdiagonal.
}

the received signal powers change over time, and the power of every signal is different from each other, as assumed in [34]. However, such assumption is unrealistic in practice. It is possible that the rank of the equivalent device signal matrix $\mathbf{D}$ is smaller than the number of devices $K$, i.e., $\operatorname{Rank}(\mathbf{D})<K$, which behaves as if some of the received signals are coherent, leading to incorrect channel estimation. Prevent possible coherent signals, we propose a novel approach in the next section to construct a signal tensor model with suitable $n$-ranks in all modes. This allows us to estimate the 2-D DoAs of $K$ devices.

\section{SPATIAL SMOOTHING-BASED TENSOR $n$-RANK ENHANCEMENT}

In this section, we analyze the relationship between the rank of $\mathbf{D}$ and the $n$-rank of $\mathcal{Y}_{\text {df }}$. We propose a spatial smoothingbased method to enhance the $n$-rank of $\mathcal{Y}_{\mathrm{df}}$. By using the proposed method, we verify that one can build a signal tensor model that provides a large enough rank in each mode to perform the DoA estimation of $K$ devices, even when the received signal powers of all the devices are equal. These powers are steady temporally across all time frames.

As discussed in Section III-C, the rank of $\mathbf{D}$ in (34) is typically smaller than the number of devices, $K$, in practice. Based on the uniqueness condition of tensor CP decomposition [35], we first provide the following theorem to evaluate the impact of $\operatorname{Rank}(\mathbf{D})$ on the $n$-ranks of the tensor $\mathcal{Y}_{\mathrm{df}}$.

Theorem 2. For $\mathcal{Y}_{d f}=\llbracket \mathcal{Z}_{d f} ; \mathbf{A}_{d f v}, \mathbf{A}_{d f h}, \mathbf{D} \rrbracket+\mathcal{N}_{d f}$, if $\operatorname{Rank}(\mathbf{D})<K$, the ranks of the signal spaces of $\mathcal{Y}_{d f}$ in all modes are smaller than the number of devices $K$, i.e., $\operatorname{Rank}\left(\mathbf{U}_{v, n}\right)<K, n=1,2,3$, where $\mathbf{U}_{v, n}$ is the mode- $n$ signal subspace of $\mathcal{Y}_{d f}$ with $\mathbf{U}_{v, 1} \in \mathbb{C}^{N_{v d c} \times K}, \mathbf{U}_{v, 2} \in \mathbb{C}^{N_{h d c} \times K}$, and $\mathbf{U}_{v, 3} \in \mathbb{C}^{M_{t} \times K}$.

\section{Proof. See Appendix II.}

According to Theorem 2, when the rank of $\mathbf{D}$ in (34) is smaller than the number of devices $K$, we cannot decompose the tensor model (32) into the signal and noise spaces in all modes. As a result, the subspace-based algorithms cannot be used to estimate the angles of the devices. To enhance the $n$ rank of the signal tensor model, we apply spatial smoothing techniques [36] to build up a sample tensor model whose signal subspace is full rank in each mode.

We divide $\mathcal{Y}_{\mathrm{df}}$ in (32) into $N_{\text {is }}$ identical subtensors in its first mode, as shown in the left-hand side of Fig. 6. The $n_{\text {is }}$-th subtensor $\left(n_{\text {is }}=1,2, \ldots, N_{\text {is }}\right)$ can be constructed as

$$
\mathcal{Y}_{\mathrm{ss}}^{\left(n_{\mathrm{is}}\right)}=\mathcal{Y}_{\mathrm{df}} \times_{1} \mathbf{J}_{\mathrm{ss}, n_{\mathrm{is}}} \in \mathbb{C}^{N_{\mathrm{ss}} \times N_{\mathrm{hdc}} \times M_{\mathrm{t}}},
$$

where $\mathbf{J}_{\mathrm{ss}, n_{\text {is }}}=\left[\mathbf{0}_{N_{\mathrm{ss}} \times\left(n_{\text {is }}-1\right)}, \mathbf{I}_{N_{\mathrm{ss}}}, \mathbf{0}_{N_{\mathrm{ss}} \times\left(N_{\text {is }}-n_{\text {is }}\right)}\right]$ and $N_{\mathrm{ss}}=$ $2 N_{\mathrm{vd}} N_{\mathrm{vs}}-N_{\text {is }}$.

We can see that

$$
\mathcal{Y}_{\mathrm{ss}}^{\left(n_{\mathrm{is}}\right)}=\llbracket \mathcal{Z}_{\mathrm{df}} ; \mathbf{A}_{\mathrm{dfv}}^{\left(n_{\mathrm{is}}\right)}, \mathbf{A}_{\mathrm{dfh}}, \mathbf{D} \rrbracket+\mathcal{N}^{\left(n_{\mathrm{is}}\right)}
$$

where $\mathbf{A}_{\mathrm{dfv}}^{\left(n_{\text {is }}\right)}=\mathbf{J}_{\mathrm{ss}, n_{\mathrm{is}}} \mathbf{A}_{\mathrm{dfv}}=\mathbf{A}_{\mathrm{dfv}}^{(1)} \mathbf{Q}_{\mathrm{ss}}^{n_{\mathrm{ss}}-1} \in \mathbb{C}^{N_{\mathrm{ss}} \times K}, \mathbf{Q}_{\mathrm{ss}}=$ $\operatorname{diag}\left(q_{\mathrm{ss}, 1}, q_{\mathrm{ss}, 2}, \ldots, q_{\mathrm{ss}, K}\right) \in \mathbb{C}^{K \times K}, q_{\mathrm{ss}, k}=e^{j \frac{2 \pi}{\lambda} h \cos \left(\theta_{k}\right)}$, and $\mathcal{N}^{\left(n_{\text {is }}\right)}=\mathcal{N}_{\mathrm{df}} \times{ }_{1} \mathbf{J}_{\mathrm{ss}, n_{\text {is }}} \in \mathbb{C}^{N_{\mathrm{ss}} \times N_{\mathrm{hdc}} \times M_{\mathrm{t}}}$ is the selected 

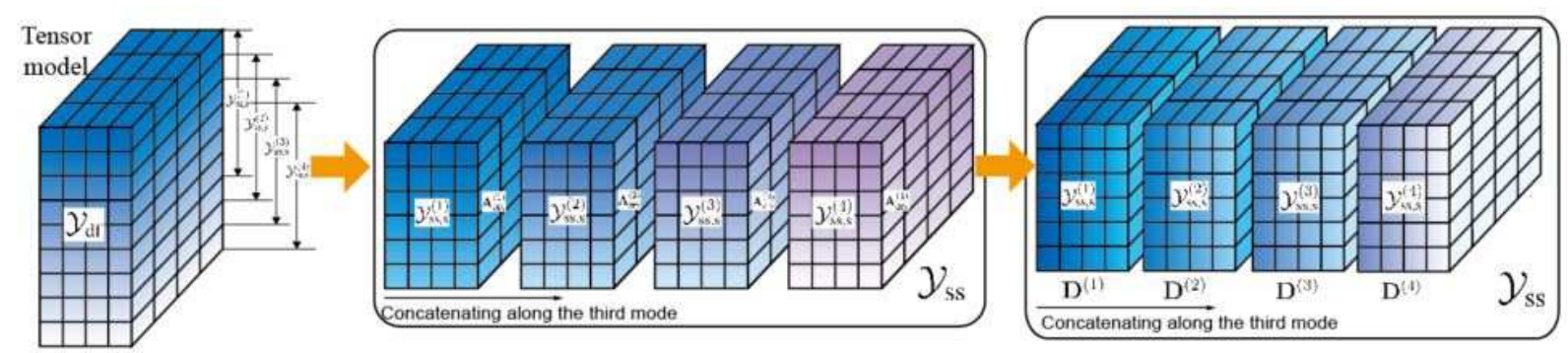

Fig. 6. An illustration of the proposed tensor $n$-rank enhancement method.

subtensor of the noise model. We can verify that only when $N_{\mathrm{vd}} N_{\mathrm{vs}}-N_{\mathrm{ss}}+1 \leq n_{\text {is }} \leq N_{\mathrm{vd}} N_{\mathrm{vs}}, \mathcal{N}^{\left(n_{\text {is }}\right)}$ has $\sigma_{\mathrm{n}}^{2}$ at the $n_{\mathrm{ss}}=\left(N_{\mathrm{vd}} N_{\mathrm{vs}}-n_{\text {is }}+1\right)$-th entry of the first mode, while $n_{\mathrm{hdc}}=P+1$ and $m_{\mathrm{t}}=1,2, \ldots, M_{\mathrm{t}}$. In all other cases, $\mathcal{N}^{\left(n_{\text {is }}\right)}=0$.

By concatenating the $N_{\text {is }}$ identical subtensors $\mathcal{Y}_{\mathrm{ss}}^{\left(n_{\mathrm{is}}\right)}, n_{\text {is }}=$ $1, \ldots, N_{\text {is }}$, as shown in the middle block of Fig. 6, the spatially smoothed signal tensor model can be constructed as

$$
\mathcal{Y}_{\mathrm{ss}}=\left[\begin{array}{cc}
\sqcup_{3} \\
n_{\mathrm{is}}=1, \ldots, N_{\mathrm{is}}
\end{array} \mathcal{Y}_{\mathrm{ss}}^{\left(n_{\mathrm{is}}\right)}\right] \in \mathbb{C}^{N_{\mathrm{ss}} \times N_{\mathrm{hdc}} \times\left(M_{\mathrm{t}} N_{\mathrm{is}}\right)},
$$

which has a rank large enough in each mode to perform DoA estimation of the $K$ devices.

Now, we proceed to verify the $n$-ranks of $\mathcal{Y}_{\text {ss. }}$. Define $\mathcal{Y}_{\mathrm{ss}, \mathrm{s}}^{\left(n_{\mathrm{s}}\right)}=\llbracket \mathcal{Z}_{\mathrm{df}} ; \mathbf{A}_{\mathrm{dfv}}^{\left(n_{\mathrm{is}}\right)}, \mathbf{A}_{\mathrm{dfh}}, \mathbf{D} \rrbracket$. We have

$$
\begin{aligned}
& {\left[\mathcal{Y}_{\mathrm{ss}, \mathrm{s}}^{\left(n_{\mathrm{is}}\right)}\right]_{n_{\mathrm{ss}}, n_{\mathrm{hdc}}, m_{\mathrm{t}}}=\sum_{k=1}^{K}\left[\mathbf{A}_{\mathrm{dfv}}^{\left(n_{\mathrm{is}}\right)}\right]_{n_{\mathrm{ss}}, k}\left[\mathbf{A}_{\mathrm{dfh}}\right]_{n_{\mathrm{hdc}}, k}[\mathbf{D}]_{m_{\mathrm{t}}, k}} \\
& =\sum_{k=1}^{K}\left(\left[\mathbf{A}_{\mathrm{dfv}}^{(1)}\right]_{n_{\mathrm{ss}}, k} q_{\mathrm{ss}, k}^{n_{\mathrm{is}}-1}\right)\left[\mathbf{A}_{\mathrm{dfh}}\right]_{n_{\mathrm{hdc}}, k}[\mathbf{D}]_{m_{\mathrm{t}}, k} \\
& =\sum_{k=1}^{K}\left[\mathbf{A}_{\mathrm{dfv}}^{(1)}\right]_{n_{\mathrm{ss}}, k}\left[\mathbf{A}_{\mathrm{dfh}}\right]_{n_{\mathrm{hdc}}, k}\left([\mathbf{D}]_{m_{\mathrm{t}}, k} q_{\mathrm{ss}, k}^{n_{\mathrm{is}}-1}\right)
\end{aligned}
$$

Hence,

$$
\mathcal{Y}_{\mathrm{ss}, \mathrm{s}}^{\left(n_{\mathrm{is}}\right)}=\llbracket \mathcal{Z}_{\mathrm{df}} ; \mathbf{A}_{\mathrm{dfv}}^{\left(n_{\mathrm{is}}\right)}, \mathbf{A}_{\mathrm{dfh}}, \mathbf{D} \rrbracket=\llbracket \mathcal{Z}_{\mathrm{df}} ; \mathbf{A}_{\mathrm{dfv} 0}, \mathbf{A}_{\mathrm{dfh}}, \mathbf{D}^{\left(n_{\mathrm{is}}\right)} \rrbracket,
$$

where $\mathbf{A}_{\mathrm{dfv} 0}=\mathbf{A}_{\mathrm{dfv}}^{(1)}$ and $\mathbf{D}^{\left(n_{\mathrm{s}}\right)}=\mathbf{D} \mathbf{Q}_{\mathrm{ss}}^{n_{\text {is }}-1}$. Therefore, 37) can be rewritten as

$$
\mathcal{Y}_{\mathrm{ss}}=\left[\underset{n_{\mathrm{is}}=1, \ldots, N_{\mathrm{is}}}{\sqcup_{3}} \mathcal{Y}_{\mathrm{ss}}^{\left(n_{\mathrm{is}}\right)}\right]=\llbracket \mathcal{Z}_{\mathrm{df}} ; \mathbf{A}_{\mathrm{dfv} 0}, \mathbf{A}_{\mathrm{dfh}}, \mathbf{D}_{\mathrm{ss}} \rrbracket+\mathcal{N}_{\mathrm{ss}},
$$

where $\mathbf{D}_{\mathrm{ss}}=\left[\left(\mathbf{D}^{(1)}\right)^{T},\left(\mathbf{D}^{(2)}\right)^{T}, \ldots,\left(\mathbf{D}^{\left(N_{\text {is }}\right)}\right)^{T}\right]^{T} \in$

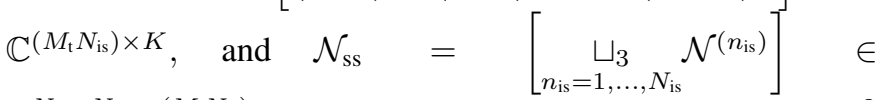
$\mathbb{C}^{N_{\mathrm{ss}} \times N_{\mathrm{hdc}} \times\left(M_{\mathrm{t}} N_{\mathrm{is}}\right)}$ is a tensor of all zeros except $\sigma_{\mathrm{n}}^{2}$ at $\left(n_{\mathrm{ss}}, 0, \tilde{m}_{\mathrm{t}}\right)$, where $n_{\mathrm{ss}}=\left(N_{\mathrm{vd}} N_{\mathrm{vs}}-n_{\text {is }}+1\right)$, $M_{\mathrm{t}}\left(n_{\text {is }}-1\right) \leq \tilde{m}_{\mathrm{t}} \leq M_{\mathrm{t}} n_{\text {is }}$, and $n_{\text {is }}=1, \ldots, N_{\text {is }}$.

An illustration of (40) is shown at the right of Fig. 6 where the recurrence relations among the divided subtensors in the mode-1 is equivalent to those in the mode- $3 . \mathbf{A}_{\mathrm{dfv}}^{\left(n_{\text {is }}\right)}$ and $\mathbf{D}^{\left(n_{\text {is }}\right)}$ are the factor matrices [25] of mode- 1 and mode-3, respectively. This property can be used to enhance the $n$-ranks of the signal tensor model.

We consider the extreme case where the received powers of all the devices are equal, and these powers are steady temporally across all time frames, i.e., $\sigma_{\mathrm{s}, m_{\mathrm{t}}, k}^{2}=\sigma_{\mathrm{s}}^{2}, m_{\mathrm{t}}=$ $1,2, \ldots, M_{\mathrm{t}}$, and $k=1,2, \ldots, K$. Then, $\mathbf{D}=\sigma_{\mathrm{s}}^{2} \mathbf{1}_{M_{\mathrm{t}} \times K}$. As a result, $\mathbf{D}_{\mathrm{ss}}$ can be rewritten as $\mathbf{D}_{\mathrm{ss}}=\sigma_{\mathrm{s}}^{2} \tilde{\mathbf{Q}}_{\mathrm{ss}} \otimes \mathbf{1}_{M_{\mathrm{t}}}$, where $\tilde{\mathbf{Q}}_{\mathrm{ss}}=\left[\mathbf{1}_{K}, \mathbf{q}_{\mathrm{ss}, 1}, \mathbf{q}_{\mathrm{ss}, 2}, \ldots, \mathbf{q}_{\mathrm{ss}, N_{\mathrm{is}}-1}\right]^{T} \in \mathbb{C}^{N_{\mathrm{is}} \times K}$, $\mathbf{q}_{\mathrm{ss}, n_{\mathrm{is}}^{\prime}}=\left[q_{\mathrm{ss}, 1}^{n_{\mathrm{s}}^{\prime}}, q_{\mathrm{ss}, 2}^{n_{\mathrm{is}}^{\prime}}, \ldots, q_{\mathrm{ss}, K}^{n_{\mathrm{is}}^{\prime}}\right]^{T}$, and $n_{\mathrm{is}}^{\prime}=1,2, \ldots, N_{\mathrm{is}}-1$.

Because the paths are from different directions, $\tilde{\mathbf{Q}}_{\mathrm{ss}}$ is an $N_{\text {is }} \times K$ Vandermonde matrix and $\operatorname{Rank}\left(\tilde{\mathbf{Q}}_{\mathrm{ss}}\right)=\min \left(N_{\mathrm{is}}, K\right)$. $\operatorname{Rank}\left(\tilde{\mathbf{Q}}_{\mathrm{ss}}\right)=K$ iff $N_{\text {is }} \geq K$. According to Lemma 1, $\operatorname{Rank}_{n}\left(\tilde{\mathcal{Y}}_{\mathrm{ss}}\right)=K$, when $\operatorname{Rank}\left(\mathbf{D}_{\mathrm{ss}}\right)=K$. Thus, the signal and noise spaces of $\mathcal{Y}_{\mathrm{ss}}$ in (37) can be decomposed in each mode.

We note that the number of $\mathcal{Y}_{\mathrm{ss}}^{\left(n_{\mathrm{s}}\right)}$ needs to be larger than the number of devices, i.e., $N_{\text {is }} \geq K$, to guarantee that $\mathcal{Y}_{\text {ss }}$ is full rank. Also, the system DoF available after spatial smoothing is proportional to the size of $\mathcal{Y}_{\mathrm{ss}}^{\left(n_{\mathrm{is}}\right)}$. Since the total number of elements in $\mathcal{Y}_{\mathrm{df}}$ is constant, increasing the number of $\mathcal{Y}_{\mathrm{ss}}^{\left(n_{\mathrm{is}}\right)}$ implies that the size of each $\mathcal{Y}_{\mathrm{ss}}^{\left(n_{\mathrm{is}}\right)}$ is smaller, while a larger size of each $\mathcal{Y}_{\mathrm{ss}}^{\left(n_{\mathrm{is}}\right)}$ means there is a smaller number of recurrence shifts available. In this sense, the best strategy is to minimize the difference between $N_{\text {is }}$ and $N_{\text {ss. }}$. Since in our system, we have $N_{\text {is }}+N_{\mathrm{ss}}-1=N_{\mathrm{vdc}}=2 N_{\mathrm{vd}} N_{\mathrm{vs}}-1$, we set $N_{\mathrm{ss}}=N_{\text {is }}=N_{\mathrm{vd}} N_{\mathrm{vs}}$.

Remark: After spatial smoothing, the system DoF becomes half of that in (18), because we divide $\mathcal{Y}_{\text {df }}$ into multiple $\mathcal{Y}_{\mathrm{ss}}^{\left(n_{\text {is }}\right)}$. Therefore, to prevent the system DoF from decreasing and achieve the target set in Section III-C, we modify (C1) in the optimization problem (18) to $N_{\mathrm{vd}} N_{\mathrm{vs}} \geq M_{\mathrm{vr}}$. Applying the analytical strategy developed in Section III-C, we formulate the modified optimization problem (18) as

$$
\begin{gathered}
\min _{N_{\mathrm{vd}}, N_{\mathrm{hd}} \in \mathbb{Z}^{+}} M_{\mathrm{rf}}=N_{\mathrm{vd}} N_{\mathrm{hd}}+\left\lceil\frac{M_{\mathrm{vr}}}{N_{\mathrm{vd}}}\right\rceil\left\lceil\frac{M_{\mathrm{hr}}}{N_{\mathrm{hd}}}\right\rceil \\
\text { s.t. (C3) and (C4). }
\end{gathered}
$$

We see that the minimum $M_{\mathrm{rf}}$ can be obtained when $N_{\mathrm{vd}} N_{\mathrm{hd}}$ and $\left\lceil M_{\mathrm{vr}} / N_{\mathrm{vd}}\right\rceil\left\lceil M_{\mathrm{hr}} / N_{\mathrm{hd}}\right\rceil$ are close or equal. Because $N_{\text {vd }}, N_{\text {hd }}, N_{\text {vs }}, N_{\text {hs }} \in \mathbb{Z}^{+}$, the optimal value of $N_{\text {vd }}, N_{\text {hd }}, N_{\text {vs }}$, and $N_{\text {hs }}$ can be obtained. 


\section{2-D DOA ESTIMATION}

In this section, the 2-D DoAs are estimated by developing a new tensor-based subspace estimation algorithm. By exploiting the recurrence relations among the UCAs, the elevation DoAs are estimated first, and then the corresponding azimuth angles are estimated by using the tensor MUSIC. The hardware and software complexities are analyzed in the end.

\section{A. Estimation of Elevation Angle}

We first propose a tensor-based total least-squares (TLS)ESPRIT algorithm to estimate the elevation angle of each device. The HOSVD of the measurement tensor $\mathcal{Y}_{\mathrm{ss}}$ is given by

$$
\begin{aligned}
\mathcal{Y}_{\mathrm{ss}} & =\mathcal{L} \times{ }_{1} \mathbf{U}_{\mathrm{dfv} 0} \times{ }_{2} \mathbf{U}_{\mathrm{dfh}} \times{ }_{3} \mathbf{U}_{\mathrm{ss}} \\
& =\llbracket \mathcal{L} ; \mathbf{U}_{\mathrm{dfv} 0}, \mathbf{U}_{\mathrm{dfh}}, \mathbf{U}_{\mathrm{ss}} \rrbracket \in \mathbb{C}^{N_{\mathrm{ss}} \times N_{\mathrm{hdc}} \times\left(M_{\mathrm{t}} N_{\mathrm{is}}\right)},
\end{aligned}
$$

where the unitary matrices, $\mathbf{U}_{\mathrm{dfv} 0} \in \mathbb{C}^{N_{\mathrm{ss}} \times N_{\mathrm{ss}}}, \mathbf{U}_{\mathrm{dfh}} \in$ $\mathbb{C}^{N_{\text {hdc }} \times N_{\text {hdc }}}$, and $\mathbf{U}_{\text {ss }} \in \mathbb{C}^{\left(M_{\mathrm{t}} N_{\text {is }}\right) \times\left(M_{\mathrm{t}} N_{\text {is }}\right)}$, are the left singular matrices of the mode- $n$ unfoldings of tensor $\mathcal{Y}_{\mathrm{ss}}$, and the core tensor $\mathcal{L} \in \mathbb{C}^{N_{\text {ss }} \times N_{\text {hdc }} \times\left(M_{\mathrm{t}} N_{\text {is }}\right)}$ is obtained by moving the singular matrices to the left-hand side of (42):

$$
\mathcal{L}=\mathcal{Y}_{\mathrm{ss}} \times{ }_{1} \mathbf{U}_{\mathrm{dfv} 0}^{H} \times{ }_{2} \mathbf{U}_{\mathrm{dfh}}^{H} \times_{3} \mathbf{U}_{\mathrm{ss}}^{H} .
$$

Define $\tilde{\mathcal{Y}}_{\mathrm{ss}}=\llbracket \mathcal{Z}_{\mathrm{df}} ; \mathbf{A}_{\mathrm{dfv} 0}, \mathbf{A}_{\mathrm{dfh}}, \mathbf{D}_{\mathrm{ss}} \rrbracket$, which contains the noise-free components of $\mathcal{Y}_{\text {ss. }}$. By removing the noise subspace component in each mode, we obtain the HOSVD model of $\tilde{\mathcal{Y}}_{\mathrm{ss}}$, as given by

$$
\tilde{\mathcal{Y}}_{\mathrm{ss}}=\mathcal{L}_{\mathrm{ss}} \times{ }_{1} \mathbf{U}_{\mathrm{dfv} 0, \mathrm{~s}} \times{ }_{2} \mathbf{U}_{\mathrm{dfh}, \mathrm{s}} \times{ }_{3} \mathbf{U}_{\mathrm{ss}, \mathrm{s}} \in \mathbb{C}^{N_{\mathrm{ss}} \times N_{\mathrm{hdc}} \times\left(M_{\mathrm{t}} N_{\mathrm{is}}\right)},
$$

where $\mathbf{U}_{\mathrm{dfv} 0, \mathrm{~s}} \in \mathbb{C}^{N_{\mathrm{ss}} \times K}, \mathbf{U}_{\mathrm{dfh}, \mathrm{s}} \in \mathbb{C}^{N_{\mathrm{hdc}} \times K}$, and $\mathbf{U}_{\mathrm{ss}, \mathrm{s}} \in$ $\mathbb{C}^{\left(M_{\mathrm{t}} N_{\mathrm{is}}\right) \times K}$ are the signal subspaces in the first, second, and third modes, respectively; and $\mathcal{L}_{\mathrm{ss}} \in \mathbb{C}^{K \times K \times K}$ is obtained by discarding insignificant singular values of $\mathcal{Y}_{\mathrm{ss}}$ in all the modes.

Define the signal subspace as

$$
\mathcal{U}_{\mathrm{s}}=\mathcal{L}_{\mathrm{ss}} \times{ }_{1} \mathbf{U}_{\mathrm{dfv} 0, \mathrm{~s}} \times{ }_{2} \mathbf{U}_{\mathrm{dfh}, \mathrm{s}} \in \mathbb{C}^{N_{\mathrm{ss}} \times N_{\mathrm{hdc}} \times K} .
$$

Because $\tilde{\mathcal{Y}}_{\text {ss }}$ can be rewritten as $\tilde{\mathcal{Y}}_{\mathrm{ss}}=\mathcal{A}_{\mathrm{ss}} \times{ }_{3} \mathbf{D}_{\mathrm{ss}}$ with $\mathcal{A}_{\mathrm{ss}}=$ $\mathcal{Z}_{\text {df }} \times{ }_{1} \mathbf{A}_{\text {dfv } 0} \times{ }_{2} \mathbf{A}_{\text {dfh }}$, we obtain

$$
\mathcal{A}_{\mathrm{ss}}=\mathcal{U}_{\mathrm{s}} \times_{3} \mathbf{D}_{\mathrm{ss}} .
$$

where $\mathbf{D}_{\mathrm{ss}} \in \mathbb{C}^{\left(M_{\mathrm{t}} N_{\mathrm{is}}\right) \times K}$ is a full column rank matrix. According to the shift-invariance relation among the subtensors in mode-1, we have

$$
\mathcal{A}_{\mathrm{ss}} \times{ }_{1} \mathbf{J}_{\mathrm{v} 2}=\mathcal{A}_{\mathrm{ss}} \times{ }_{1} \mathbf{J}_{\mathrm{v} 1} \times{ }_{3} \Theta_{\mathrm{v}}
$$

where $\boldsymbol{\Theta}_{\mathrm{v}}=\operatorname{diag}\left(e^{-j \frac{2 \pi}{\lambda} h \cos \left(\theta_{1}\right)}, \ldots, e^{-j \frac{2 \pi}{\lambda} h \cos \left(\theta_{K}\right)}\right), \mathbf{J}_{\mathrm{v} 1}=$ $\left[\mathbf{I}_{M_{\mathrm{vr}}-1}, \mathbf{0}_{\left(M_{\mathrm{vr}}-1\right) \times 1}\right]$, and $\mathbf{J}_{\mathrm{v} 2}=\left[\mathbf{0}_{\left(M_{\mathrm{vr}}-1\right) \times 1}, \mathbf{I}_{M_{\mathrm{vr}}-1}\right]$. Let

$$
\mathcal{U}_{\mathrm{sv} 1}=\mathcal{U}_{\mathrm{s}} \times 1 \mathbf{J}_{\mathrm{v} 1} \text { and } \mathcal{U}_{\mathrm{sv} 2}=\mathcal{U}_{\mathrm{s}} \times{ }_{1} \mathbf{J}_{\mathrm{v} 2} .
$$

By substituting (46) into (47), we have $\mathcal{U}_{\mathrm{sv} 2}=\mathcal{U}_{\mathrm{sv} 1} \times_{3} \boldsymbol{\Psi}_{\mathrm{v}}$, where $\boldsymbol{\Psi}_{\mathrm{v}} \in \mathbb{C}^{K \times K}$ is a full rank matrix. To obtain the estimate of $\boldsymbol{\Psi}_{\mathrm{v}}$, we define $\boldsymbol{\Upsilon}_{\mathrm{v}}=\left[\begin{array}{ll}\boldsymbol{\Upsilon}_{\mathrm{v} 1} & \boldsymbol{\Upsilon}_{\mathrm{v} 2}\end{array}\right] \in \mathbb{C}^{K \times 2 K}$. We now generalize the matrix TLS problem formulation [31] to the tensor setting, as follows.

$$
\begin{aligned}
\hat{\boldsymbol{\Upsilon}}_{\mathrm{v}}=\arg \min _{\boldsymbol{\Upsilon}_{\mathrm{v}}}\left\|\mathcal{U}_{\mathrm{sv} 1} \times{ }_{3} \boldsymbol{\Upsilon}_{\mathrm{v} 1}+\mathcal{U}_{\mathrm{sv} 2} \times{ }_{3} \boldsymbol{\Upsilon}_{\mathrm{v} 2}\right\|, \\
\text { s.t. } \quad \boldsymbol{\Upsilon}_{\mathrm{v}} \boldsymbol{\Upsilon}_{\mathrm{v}}^{H}=\mathbf{I}_{K},
\end{aligned}
$$

which finds a unitary matrix $\Upsilon_{\mathrm{v}}$ with orthogonal submatrices to $\mathcal{U}_{\mathrm{sv} 1}$ and $\mathcal{U}_{\mathrm{sv} 2}$ in mode- 3 .

The mode- 3 unfoldings of $\mathcal{U}_{\mathrm{sv} 1}$ is given by

$$
\mathbf{U}_{\mathrm{sv} 1(3)}=\mathbf{U}_{\mathrm{s}(3)}\left(\mathbf{J}_{\mathrm{v} 1} \otimes \mathbf{I}_{N_{\mathrm{hdc}}}\right)^{T},
$$

where $\mathbf{U}_{\mathrm{s}(3)} \in \mathbb{C}^{K \times M_{\mathrm{vr}} M_{\mathrm{hr}} M_{\mathrm{f}}}$ is the mode-3 unfolding of $\mathcal{U}_{\mathrm{s}}$. The mode- 3 unfoldings of $\mathcal{U}_{\mathrm{sv} 2}$ can be formulated in the same way. Since $\|\mathcal{A}\|=\left\|\mathbf{A}_{(n)}\right\|_{\mathrm{F}}(n=1,2, \ldots, N)$ [25], we rewrite the tensor TLS problem (49) as

$$
\begin{gathered}
\hat{\boldsymbol{\Upsilon}}_{\mathrm{v}}=\arg \min _{\boldsymbol{\Upsilon}_{\mathrm{v}}} \| \mathbf{\Upsilon}_{\mathrm{v} 1} \mathbf{U}_{\mathrm{s}(3)}\left(\mathbf{J}_{\mathrm{v} 1} \otimes \mathbf{I}_{N_{\mathrm{hdc}}}\right)^{T} \\
\quad+\boldsymbol{\Upsilon}_{\mathrm{v} 2} \mathbf{U}_{\mathrm{s}(3)}\left(\mathbf{J}_{\mathrm{v} 2} \otimes \mathbf{I}_{N_{\mathrm{hdc}}}\right)^{T} \|_{\mathrm{F}} \\
=\arg \min _{\boldsymbol{\Upsilon}_{\mathrm{v}}}\left\|\mathbf{W}_{\mathbf{v}} \mathbf{\Upsilon}_{\mathrm{v}}^{T}\right\|_{\mathrm{F}},
\end{gathered}
$$

where

$$
\begin{aligned}
\mathbf{W}_{\mathrm{v}} & =\left[\begin{array}{ll}
\left(\mathbf{J}_{\mathrm{v} 1} \otimes \mathbf{I}_{N_{\mathrm{hdc}}}\right) \mathbf{U}_{\mathrm{s}(3)}^{T} & \left(\mathbf{J}_{\mathrm{v} 2} \otimes \mathbf{I}_{N_{\mathrm{hdc}}}\right) \mathbf{U}_{\mathrm{s}(3)}^{T}
\end{array}\right] \\
& \in \mathbb{C}^{\left(N_{\mathrm{ss}}-1\right) N_{\mathrm{hdc}} \times 2 K} .
\end{aligned}
$$

The SVD of $\mathbf{W}_{\mathrm{v}}^{H} \mathbf{W}_{\mathrm{v}}$ is written as $\mathbf{W}_{\mathrm{v}}^{H} \mathbf{W}_{\mathrm{v}}=\dot{\mathbf{U}}_{\mathrm{v}} \dot{\boldsymbol{\Lambda}}_{\mathrm{v}} \dot{\mathbf{V}}_{\mathrm{v}}$, where $\dot{\mathbf{U}}_{\mathrm{v}} \in \mathbb{C}^{2 K \times 2 K}$ and $\dot{\mathbf{V}}_{\mathrm{v}} \in \mathbb{C}^{2 K \times 2 K}$ are the left and right singular matrices, respectively; and $\dot{\Lambda}_{\mathrm{v}} \in \mathbb{C}^{2 K \times 2 K}$ contains the singular values. We partition $\dot{\mathbf{U}}_{\mathrm{v}}$ into four blocks:

$$
\dot{\mathbf{U}}_{\mathrm{v}}=\left[\begin{array}{cc}
\dot{\mathbf{U}}_{\mathrm{v} 11} & \dot{\mathbf{U}}_{\mathrm{v} 12} \\
\dot{\mathbf{U}}_{\mathrm{v} 21} & \dot{\mathbf{U}}_{\mathrm{v} 22}
\end{array}\right] \in \mathbb{C}^{2 K \times 2 K} .
$$

Let $\hat{\Upsilon}_{\mathrm{v} 1}=\dot{\mathbf{U}}_{\mathrm{v} 12}^{T} \in \mathbb{C}^{K \times K}$ and $\hat{\Upsilon}_{\mathrm{v} 2}=\dot{\mathbf{U}}_{\mathrm{v} 22}^{T} \in \mathbb{C}^{K \times K}$. According to the standard TLS [31], the estimate of $\boldsymbol{\Psi}_{\mathrm{v}}$ is given by $\hat{\mathbf{\Psi}}_{\mathrm{v}}=-\hat{\boldsymbol{\Upsilon}}_{\mathrm{v} 1} \hat{\boldsymbol{\Upsilon}}_{\mathrm{v} 2}^{-1}$, where the $K$ eigenvalues of $\hat{\mathbf{\Psi}}_{\mathrm{v}}$, i.e., $\psi_{\mathrm{v}, k}, k=1,2, \ldots, K$, are sorted in descending order. According to the array steering expression (8), the elevation angle of the $k$-th device can be estimated as

$$
\hat{\theta}_{k}=\arccos \left(j \lambda \ln \left(\psi_{\mathrm{v}, k}\right) /(2 \pi h)\right) .
$$

\section{B. Estimation of Azimuth Angle}

We use the tensor-MUSIC algorithm [22] to estimate the azimuth angle of each device. According to (44), we can discard the largest $K$ singular values of the mode- $n$ unfoldings of $\mathcal{Y}_{\mathrm{ss}}$ and obtain the noise subspace in mode-2, $\mathbf{U}_{\mathrm{dfh}, \mathrm{n}} \in$ $\mathbb{C}^{N_{\text {hdc }} \times\left(N_{\text {hdc }}-K\right)}$. Then, we generalize the matrix-based MUSIC to the tensor, and the tensor MUSIC spectrum of the azimuth angle can be defined as

$$
\operatorname{SP}_{\text {MUSIC }}(\Phi)=\left\|\mathcal{A}_{\mathrm{ss}} \times{ }_{2} \mathbf{U}_{\mathrm{dfh}, \mathrm{n}}^{H}\right\|^{-2}
$$

where $\Phi=\left[\phi_{1}, \phi_{2}, \ldots, \phi_{K}\right]$. The mode-2 unfolding of $\mathcal{A}_{\mathrm{ss}}$ can be expressed as

$$
\mathbf{A}_{\mathrm{ss}(2)}=\mathbf{A}_{\mathrm{dfh}} \mathbf{Z}_{\mathrm{df}(2)}\left(\mathbf{I}_{M_{\mathrm{t}} N_{\mathrm{is}}} \otimes \mathbf{A}_{\mathrm{dfv} 0}\right)^{T},
$$



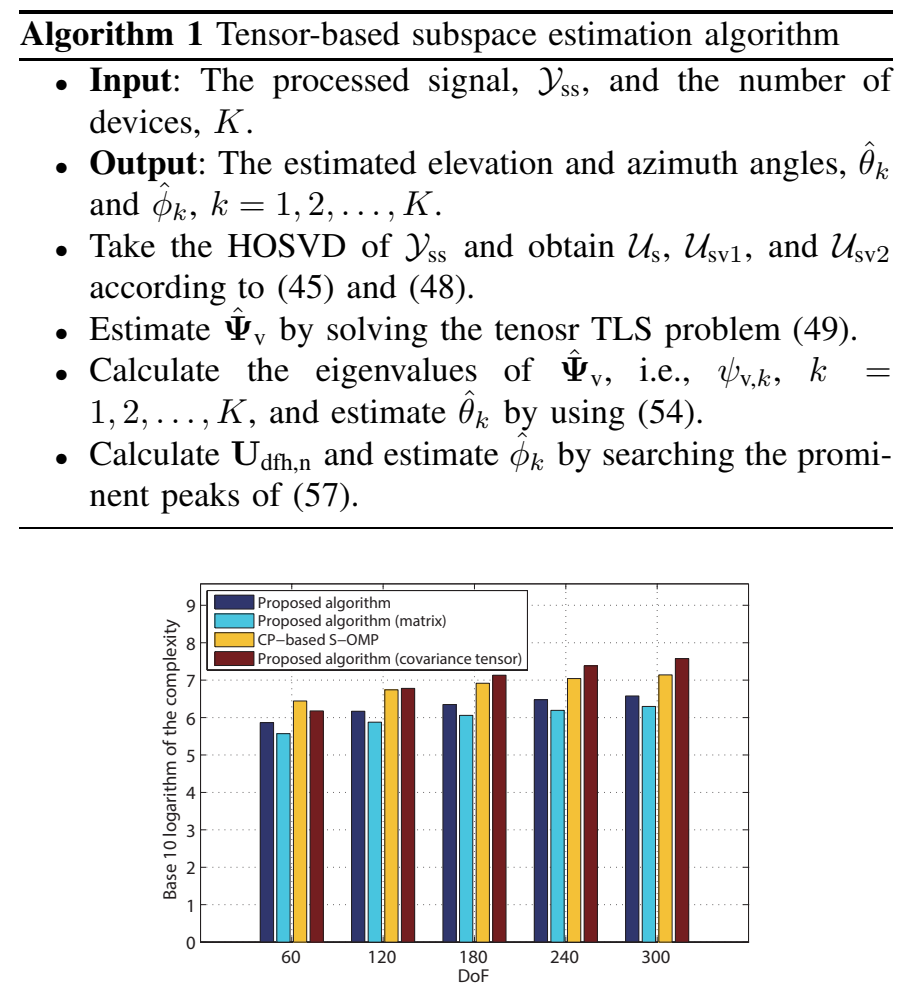

Fig. 7. Variation of the software complexity vs. DoF.

where $\mathbf{Z}_{\mathrm{df}(2)}$ is the mode- 2 unfolding of $\mathcal{Z}_{\mathrm{df}}$. According to a property of tensor multiplication and unfolding: $\|\mathcal{A}\|=$ $\left\|\mathbf{A}_{(n)}\right\|_{\mathrm{F}}, n=1,2, \ldots, N$, we can rewrite the tensor MUSIC spectrum (55) as

$$
\operatorname{SP}_{\text {MUSIC }}(\Phi)=\left\|\mathbf{U}_{\mathrm{dfh}, \mathrm{n}}^{H} \mathbf{A}_{\mathrm{dfh}} \mathbf{Z}_{\mathrm{df}(2)}\left(\mathbf{I}_{M_{\mathrm{t}} N_{\mathrm{is}}} \otimes \mathbf{A}_{\mathrm{dfv} 0}\right)^{T}\right\|_{\mathrm{F}}^{-2} .
$$

By substituting the estimated elevation angle of each device (54) into (57), the corresponding azimuth angle $\phi_{k}$ can be estimated by searching the prominent peaks of the tensor MUSIC spectrum (57). Algorithm 1 summarizes the procedure of the proposed tensor-based subspace estimation algorithm.

\section{Complexity Analysis}

We analyze the hardware and software complexity of the proposed tensor-based parameter estimation algorithm.

For the hardware complexity, the use of the proposed hybrid array reduces the hardware complexity to $O\left(M_{\mathrm{rf}}\right)=$ $O\left(N_{\mathrm{vd}} N_{\text {hd }}+N_{\mathrm{vs}} N_{\mathrm{hs}}\right)$, while a fully digital array using the same number of antennas would have a hardware complexity of $O\left(M_{\mathrm{bs}}\right)$. We compare the system power consumption between our system, and the systems using hourglass arrays [28] and OBAs [32 9 . According to [37], the power of a hybrid array is consumed by its RF chains, analog-to-digital converters (ADCs), local oscillators, power amplifiers, and phase shifters. Since the proposed method, and the methods using hourglass arrays and OBAs, are only different in terms of the design

\footnotetext{
${ }^{9}$ For a fair comparison, all these systems do not consider using spatial smoothing, and the periodicity of UCAs is considered here.
}

of RF connection matrices, the numbers of required phase shifters, local oscillators, and power amplifiers are the same across these three methods. As a result, the difference of system power consumption between the methods depends on the numbers of RF chains and ADSs. Also note that the number of RF chains is equal to the number of ADCs. Assume that the dimension of phase-shifter output ports is $M_{\mathrm{hr}} \times M_{\mathrm{vr}}=29 \times 17$. In our system, only $M_{\mathrm{rf}}=N_{\mathrm{vd}} N_{\mathrm{hd}}+N_{\mathrm{vs}} N_{\mathrm{hs}}-1=32 \mathrm{RF}$ chains (and ADSs) are required by solving (18). However, in the systems using hourglass arrays and OBAs, the numbers of required RF chains are 37 and 35, respectively.

As for the signal processing complexity, we compare the computational complexity of the proposed tensor-based algorithm with its matrix-based counterpart, which formulates the signal model in the matrix form and uses matrix-based ESPRIT-MUSIC algorithm for DoA estimation. For matrixbased algorithms, the computational complexity of performing SVD to the measurement sample matrix and truncating its rank to $K$ is $O\left(N_{\mathrm{ss}} N_{\text {hdc }} M_{\mathrm{t}} N_{\text {is }} K\right)$. The complexities of estimating the elevation and azimuth angles are $O\left(K^{3}+N_{\mathrm{ss}} N_{\mathrm{hdc}}\right)$ and $O\left(N_{\text {hdc }} K^{2}+N_{\text {hdc }}^{2} K D\right)$, respectively. $D$ is the size of search dimension. For the proposed tensor-based algorithm, the computational complexity of taking the HOSVD of the tensor model is $O\left(N_{\mathrm{ss}} N_{\mathrm{hdc}} M_{\mathrm{t}} N_{\mathrm{is}} K\right)$. The computational complexities of estimating elevation and azimuth angles are $O\left(N_{\mathrm{ss}} N_{\mathrm{hdc}}+K^{3}\right)$ and $O\left(N_{\text {ss }} N_{\text {hdc }} M_{\mathrm{t}} N_{\text {is }} K+N_{\text {hdc }}^{2} K D\right)$, respectively. The new tensor-based algorithm needs slightly more computations, but is in the same order as its matrix-based counterpart.

We also compare our algorithm with the CP-based simultaneous-orthogonal matching pursuit (S-OMP) algorithm [22]. The algorithm first applies CP decomposition to decompose the received signal tensor model, and then applies S-OMP to estimate the parameters. The complexities of the CP decomposition and S-OMP are $O\left(N_{\text {ss }} N_{\text {hdc }} M_{\mathrm{t}} N_{\text {is }} K+N_{\text {ss }} N_{\text {hdc }} K^{2}+\right.$ $\left.K^{3}\right)$ and $O\left(N_{\mathrm{ss}} N_{\mathrm{hdc}} M_{\mathrm{t}} N_{\mathrm{is}}\left(N_{1}+N_{2}\right)\right)$, respectively, where $N_{1} \gg K$ and $N_{2} \gg K$ are the dimensions of the OMP grid. The complexity of CP-based subspace algorithm is much higher than that of our HOSVD-based algorithm.

Note that all the operations in our DoA estimation algorithm are on the signal data tensor model directly. If our algorithms operate on the signal covariance tensor, we need to calculate the signal covariance tensor model [20], [21], [38]

$$
\mathcal{R}_{\mathrm{ss}}=\frac{1}{M_{\mathrm{t}} N_{\mathrm{is}}} \sum_{m=1}^{M_{\mathrm{t}} N_{\mathrm{is}}} \mathcal{Y}_{\mathrm{ss}, m} \circ \mathcal{Y}_{\mathrm{ss}, m}^{*} \in \mathbb{C}^{N_{\mathrm{ss}} \times N_{\mathrm{hdc}} \times N_{\mathrm{ss}} \times N_{\mathrm{hdc}}},
$$

where $\mathbf{Y}_{\mathrm{ss}, m} \in \mathbb{C}^{N_{\mathrm{ss}} \times N_{\mathrm{hdc}}}$ is the $m$-th subtensor of $\mathcal{Y}_{\mathrm{ss}}, m=$ $1,2, \ldots, M_{\mathrm{t}} N_{\mathrm{is}}$, and then, take the HOSVD of (58). The computational complexity of this process is $O\left(M_{\mathrm{t}} N_{\text {is }} N_{\mathrm{ss}}^{2} N_{\mathrm{hdc}}^{2}+\right.$ $\left.N_{\mathrm{ss}}^{2} N_{\text {hdc }}^{2} K\right)$, which needs much more computations than our algorithms.

The results of the computational complexities of our algorithm, its matrix-based counterpart, CP-based S-OMP algorithm, and the proposed algorithm operating on the covariance tensor, as a function of the system DoF, $O\left(N_{\text {ss }} N_{\text {hdc }}\right)$, are presented in Fig. 7, where $M_{\mathrm{t}}=20, N_{\mathrm{is}}=20, K=15$, and 

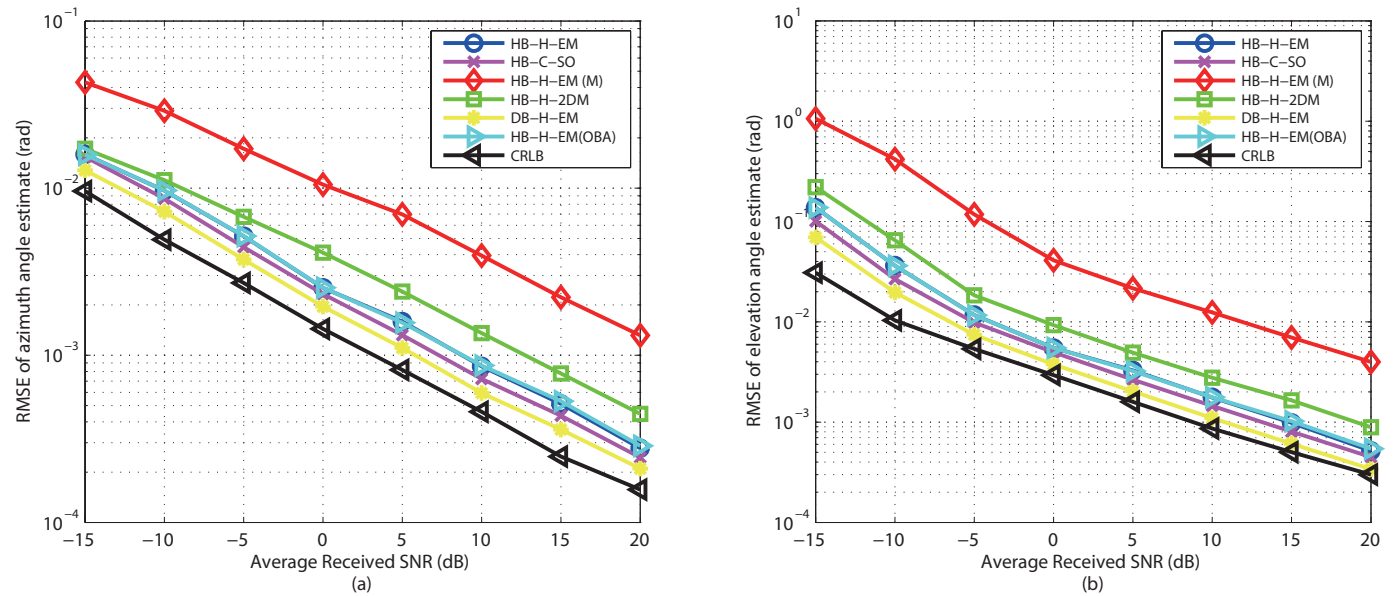

Fig. 8. RMSE vs. the average received SNR for the estimation of DoAs for identifying $K=50$ devices. (a) Azimuth angle; (b) Elevation angle.
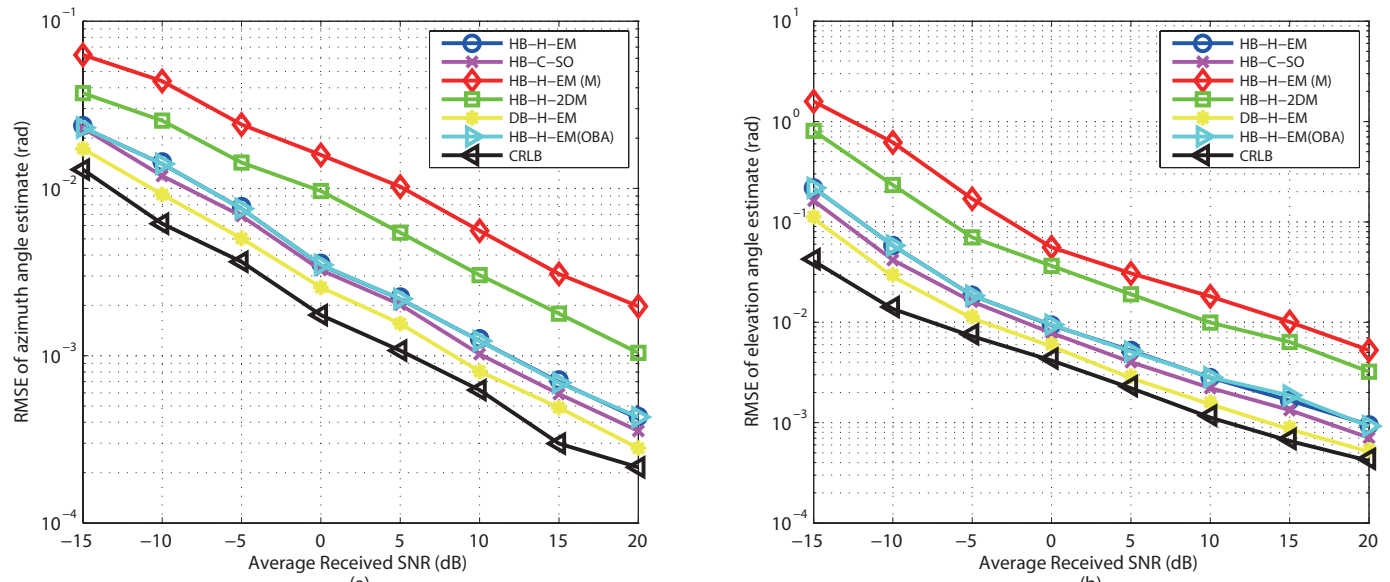

(b)

Fig. 9. RMSE vs. the average received SNR for the estimation of DoAs for identifying $K=200$ devices. (a) Azimuth angle; (b) Elevation angle.

$N_{1}=N_{2}=50$. The figure shows that our proposed algorithm requires more computations than its matrix-based counterpart at the gain of significantly improved DoA estimation performance, as will be seen in Section VI. However, compared to the other two algorithms, the computational complexity of our algorithm is much lower.

\section{Simulation Results}

In this section, simulation results are provided to demonstrate the performance of the proposed algorithm. The system bandwidth is $B=1 \mathrm{GHz}$. The number of time frames is set to $M_{\mathrm{t}}=20$. The reference radial frequency $f=28 \mathrm{GHz}$. The vertical spacing between adjacent receiving UCAs is $h=0.5 \lambda$ and the radius of the UCyA is $r=2 \lambda$, where $\lambda=c / f$ and $c$ is the speed of light. The geometry parameters of the UCyA are $M_{\mathrm{v}}=25$ and $M_{\mathrm{h}}=30$. For the hybrid beamforming, we set $N_{\mathrm{vd}}=5, N_{\mathrm{hd}}=5, N_{\mathrm{vs}}=5$, and $N_{\mathrm{hs}}=6$, so there are $M_{\mathrm{rf}}=N_{\mathrm{vd}} N_{\mathrm{hd}}+N_{\mathrm{vs}} N_{\mathrm{hs}}-1=54 \mathrm{RF}$ chains in our system.

Fig. 8 plots the root mean square errors (RMSEs) for the estimates of the azimuth and elevation angles versus the average received SNR, where the DoAs of $K=50$ devices are estimated. By using the proposed nested sparse hybrid beamforming, we compare the proposed HOSVD-based ESPRIT-MUSIC (HB-H-EM) algorithm with its reduced version in the matrix form (HB-H-EM (M)), the CP-based S-OMP (HB-C-SO) algorithm [22], the HOSVD-based 2D MUSIC (HB-H-2DM) algorithm [21], and the proposed algorithm but using OBA to design the RF connection matrix (HB-HEM (OBA)). We also apply the proposed algorithm for fully digital beamforming (DB-H-EM), and provide the Cramér-Rao lower bound (CRLB) [39] as a reference. We can see that all the estimated algorithms approach the CRLB, as the average received SNR increases. Fig. 8 also shows that our proposed tensor-based algorithm provides a better accuracy than its matrix-based counterparts. This is because the tensor-based algorithm can suppress the noise components in each mode of the signal tensor model, while the matrix-based algorithm can only suppress the noise in the time domain corresponding to the third mode in this paper. By applying CP to decompose the signal tensor model, HB-C-SO achieves better estimation performance than other HOSVD-like algorithms. However, the performance improvement is limited because HB-C-SO 


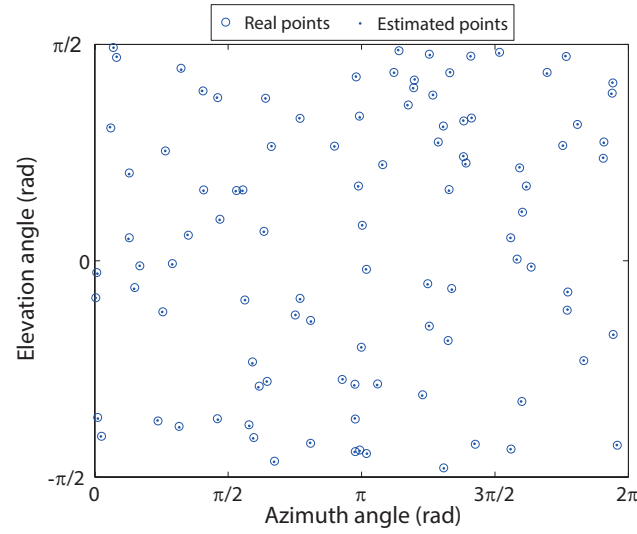

Fig. 10. 2-D DoA estimation by using the proposed algorithm for 100 devices.

uses S-OMP to estimate the parameters, generating quantized estimates only. We also observe that the precision of the angle estimation of our proposed algorithm is a bit lower than that of DB-H-EM. However, both DB-H-EM and HB-C-SO have a much higher complexity than our algorithm, as analyzed in Section V-C. In addition, the estimation accuracy is nearly the same between the proposed HB-H-EM and HB-H-EM (OBA). This is because the DoA estimation accuracy depends on the dimension of the difference coarray, not the dimension of the $\mathrm{RF}$ chain network, while the constructed difference coarrays of the two methods are identical.

Fig. 9 shows the RMSEs for the estimates of DoAs versus the average received SNR. The number of devices is $K=200$. By comparing Figs. 8 and 9 , we see that for the fixed SNR and a fixed number of RF chains, the estimation accuracy of all the tested algorithms decreases as $K$ increases. This is because as $K$ grows, more signal components need to be estimated and distinguished. Compared with Fig. 8, Fig. 9also shows that the performance degeneration of HB-H-2DM is larger than other algorithms. This is because HB-H-2DM uses signal covariance tensors for the 2-D DoA estimation, and its MUSIC spectrum is a product of multiple separable secondorder mode- $n$ spectra, which results in undesirable cross-terms [21] and compromises the estimation accuracy.

Fig. 10 evaluates the performance of our proposed algorithm. Without loss of generality, here we estimate the 2-D DoAs of $K=100$ devices, where SNR $=5 \mathrm{~dB}$. As seen from the results, the proposed algorithm can accurately estimate the azimuth and elevation angles of 100 devices. All the estimates are well matched with the actual values, while we only use $54 \mathrm{RF}$ chains in our system.

\section{CONCLUSION}

We presented a novel sparse nested hybrid UCyA for mIoT networks. By exploiting the difference coarray technique and tailoring for the UCyA, we proposed a channel estimation scheme based on the second-order statistics of the received signals. As a result, the designed hybrid array only requires a small number of RF chains to achieve DoA estimation for a massive number of IoT devices. We proposed a spatial smoothing-based method to enhance the $n$-ranks of the signal tensor model. By using the method, a large enough rank in each mode of the signal tensor model was provided for the DoA estimation of $K$ devices. Given the designed hybrid array, a new tensor-based 2-D DoA estimation algorithm was proposed, and it can significantly improve the estimation accuracy while reducing the computational complexity. Simulation results demonstrated that our proposed hybrid array system can accurately estimate the 2-D DoAs of a large number of IoT devices.

\section{APPENDIX I}

\section{PROOF OF THEOREM 1}

Let $\gamma\left(\theta_{k}\right)=2 \pi r \sin \left(\theta_{k}\right) / \lambda$. The phase-space transformation of $\mathbf{a}_{\mathbf{h}}\left(\theta_{k}, \phi_{k}\right)$ can be expressed as

$$
\begin{aligned}
& a_{\mathrm{hps}, p}\left(\theta_{k}, \phi_{k}\right)=\sum_{m_{\mathrm{h}}=1}^{M_{\mathrm{h}}}\left(a_{\mathrm{h}, m_{\mathrm{h}}}\left(\theta_{k}, \phi_{k}\right)\right) e^{-j \frac{2 \pi\left(m_{\mathrm{h}}-1\right)}{M_{\mathrm{h}}} p} \\
& =\sum_{m_{\mathrm{h}}=1}^{M_{\mathrm{h}}}\left(\frac{1}{\sqrt{M_{\mathrm{h}}}} e^{j \gamma\left(\theta_{k}\right) \cos \left(\phi_{k}-\varphi_{m_{\mathrm{h}}}\right)}\right) e^{-j \frac{2 \pi\left(m_{\mathrm{h}}-1\right)}{M_{\mathrm{h}}} p} \\
& \stackrel{(\mathrm{a})}{=} \sum_{m_{\mathrm{h}}=1}^{M_{\mathrm{h}}}\left(\frac{1}{\sqrt{M_{\mathrm{h}}}} \sum_{q=-\infty}^{\infty} j^{q} J_{q}\left(\gamma\left(\theta_{k}\right)\right) e^{j q\left(\phi_{k}-\varphi_{m_{\mathrm{h}}}\right)}\right) \\
& \times e^{-j \frac{2 \pi\left(m_{\mathrm{h}}-1\right)}{M_{\mathrm{h}}} p} \\
& \stackrel{(\mathrm{b})}{=} \frac{1}{\sqrt{M_{\mathrm{h}}}} \sum_{Q=-\infty}^{\infty} M_{\mathrm{h}} j^{\left(Q M_{\mathrm{h}}-p\right)} J_{\left(Q M_{\mathrm{h}}-p\right)}\left(\gamma\left(\theta_{k}\right)\right) \\
& \times e^{j\left(Q M_{\mathrm{h}}-p\right) \phi_{k}} \\
& \stackrel{(\mathrm{c})}{=} \sqrt{M_{\mathrm{h}}}\left[j^{p} J_{p}\left(\gamma\left(\theta_{k}\right)\right) e^{-j p \phi_{k}}\right. \\
& \left.+\sum_{Q=-\infty, Q \neq 0}^{\infty} \varepsilon_{p, Q}\left(\gamma\left(\theta_{k}\right), \phi_{k}\right)\right]
\end{aligned}
$$

where

$\varepsilon_{p, Q}\left(\gamma\left(\theta_{k}\right), \phi_{k}\right)=j^{\left(Q M_{\mathrm{h}}-p\right)} J_{\left(Q M_{\mathrm{h}}-p\right)}\left(\gamma\left(\theta_{k}\right)\right) e^{j\left(Q M_{\mathrm{h}}-p\right) \phi_{k}}$.

In (59), (a) and (c) follow the important properties of the Bessel function, i.e., $e^{j x \cos y}=\sum_{v=-\infty}^{\infty} j^{v} J_{v}(x) e^{j v y}$ and $J_{-v}(x)=(-1)^{v} J_{v}(x)$, respectively. (b) is obtained by letting $p+q=Q M_{\mathrm{h}}$ [40].

Let $x=v \rho, \rho \in(0,1)$ and $v \in \mathbb{Z}^{+}$. The Bessel function, $J_{v}(x)$, whose order $v$ exceeds its argument, $x$, can be written in the following form [40]

$$
J_{v}(v \rho)=\frac{1}{\pi} \int_{0}^{\pi} \exp (-v F(\vartheta, \rho)) d \vartheta,
$$

where

$F(\vartheta, \rho)=\log \left(\frac{\vartheta+\sqrt{\vartheta^{2}-\rho^{2} \sin ^{2} \vartheta}}{\rho \sin \vartheta}\right)-\cot \vartheta \sqrt{\vartheta^{2}-\rho^{2} \sin ^{2} \vartheta}$.

The partial derivative of (60) with respect to $\rho$ is given by 


\section{REFERENCES}

$$
\begin{aligned}
\frac{\partial}{\partial \rho} J_{v}(v \rho)= & -\frac{v}{\pi} \int_{0}^{\pi} \frac{\partial F(\vartheta, \rho)}{\partial \rho} \exp (-v F(\vartheta, \rho)) d \vartheta \\
& =\frac{v}{\pi \rho} \int_{0}^{\pi} g(\vartheta, \rho) \exp (-v F(\vartheta, \rho)) d \vartheta
\end{aligned}
$$

where $g(\vartheta, \rho)=\left(\vartheta-\rho^{2} \sin \vartheta \cos \vartheta\right) / \sqrt{\vartheta^{2}-\rho^{2} \sin ^{2} \vartheta}$. Given that

$$
\begin{aligned}
g(\vartheta, \rho) & =\frac{\vartheta-\rho^{2} \sin \vartheta \cos \vartheta}{\sqrt{\vartheta^{2}-\rho^{2} \sin ^{2} \vartheta}} \geq \frac{\vartheta-\sin \vartheta \cos \vartheta}{\sqrt{\vartheta^{2}-\rho^{2} \sin ^{2} \vartheta}} \\
& \geq \frac{\vartheta-\sin \vartheta}{\sqrt{\vartheta^{2}-\rho^{2} \sin ^{2} \vartheta}} \geq 0,
\end{aligned}
$$

we have $\partial J_{v}(v \rho) / \partial \rho>0$, and conclude that $J_{v}(v \rho)$ is an increasing function of $\rho$. Thus, $J_{v}(v \rho)<J_{v}(v)$.

On the other hand, the partial derivative of (60) with respect to $v$ is given by

$$
\frac{\partial}{\partial v} J_{v}(v \rho)=-\frac{1}{\pi} \int_{0}^{\pi} F(\vartheta, \rho) \exp (-v F(\vartheta, \rho)) d \vartheta .
$$

Because

$$
\frac{\partial}{\partial \vartheta} F(\vartheta, \rho)=\frac{(1-\rho \cot \vartheta)^{2}}{\sqrt{\vartheta^{2}-\rho^{2} \sin ^{2} \vartheta}}+\sqrt{\vartheta^{2}-\rho^{2} \sin ^{2} \vartheta} \geq 0
$$

and $\partial F(0, \rho) / \partial \rho=-\sqrt{1-\rho^{2}} / \rho \leq 0$, we have $F(\vartheta, \rho) \geq$ $F(0, \rho) \geq F(0,1)=0$ and hence, $\partial J_{v}(v \rho) / \partial v<0$. This means that $J_{v}(v \rho)$ is a decreasing function of $v$, i.e., $J_{v}(v \rho)<$ $J_{1}(\rho)$. Therefore, we have $J_{v}(v \rho)<J_{v}(v)<J_{1}(1) \approx 0.4$ with $\rho \in(0,1)$ and $v \in \mathbb{Z}^{+}$. For $|v|>|x|,\left|J_{v}(x)\right| \approx 0$ with $v \in \mathbb{Z}^{+}$. Based on this property, , both $\varepsilon_{p, Q}\left(\gamma\left(\theta_{k}\right), \phi_{k}\right)$ and $J_{p}\left(\gamma\left(\theta_{k}\right)\right)$ in (59) can be suppressed in the case of $|p|>P>$ $\gamma\left(\theta_{k}\right)$, since $P \geq\lfloor 2 \pi r / \lambda\rfloor>2$ and $M_{\mathrm{h}} \geq\lfloor 4 \pi r / \lambda\rfloor>2$. When $|p| \leq P$, we can only ignore $\varepsilon_{p, Q}\left(\gamma\left(\theta_{k}\right), \phi_{k}\right)$. Thus, (59) can be approximated by (13). This concludes the proof.

\section{APPENDIX II}

\section{PROOF OF THEOREM 2}

Define $\mathcal{Y}_{\mathrm{dfs}}=\llbracket \mathcal{Z}_{\mathrm{df}} ; \mathbf{A}_{\mathrm{dfv}}, \mathbf{A}_{\mathrm{dfh}}, \mathbf{D} \rrbracket$, which is the noisefree model of $\mathcal{Y}_{\mathrm{df}}$. Thus, $\mathcal{Y}_{\mathrm{dfs}}$ consists of all the signal space components.

Because $\mathbf{A}_{\mathrm{dfv}} \in \mathbb{C}^{N_{\mathrm{vdc}} \times K}$ and $\mathbf{A}_{\mathrm{dfh}} \in \mathbb{C}^{N_{\mathrm{hdc}} \times K}$ are Vandermonde matrices, and in our system, we have $N_{\text {vdc }} \geq K$ and $N_{\text {hdc }} \geq K$, according to uniqueness condition of the CP decomposition, the $n$-ranks of $\mathcal{Y}_{\text {dfs }}$ depends on the rank of $\mathbf{D}$.

On the other hand, the SVD of the mode- $n$ unfolding of $\mathcal{Y}_{\mathrm{dfs}}, \mathbf{Y}_{\mathrm{dfs}(n)}$, can be written as $\mathbf{Y}_{\mathrm{dfs}(n)}=\mathbf{U}_{\mathrm{vs}, n} \boldsymbol{\Sigma}_{\mathrm{vs}, n} \mathbf{V}_{\mathrm{vs}, n}^{H}$, where $n=1,2,3$, and we have $\operatorname{Rank}\left(\mathbf{Y}_{\mathrm{dfs}(n)}\right)=$ $\operatorname{Rank}\left(\mathbf{U}_{\mathrm{vs}, n}\right)=\operatorname{Rank}\left(\boldsymbol{\Sigma}_{\mathrm{vs}, n}\right)=\operatorname{Rank}\left(\mathbf{V}_{\mathrm{vs}, n}\right)$. When $\operatorname{Rank}(\mathbf{D})<K$, we have $\operatorname{Rank}\left(\mathbf{Y}_{\mathrm{dfs}(n)}\right)<K$, and thus $\operatorname{Rank}\left(\mathbf{U}_{\mathrm{v}, n}\right)<K$.

This concludes the proof of Theorem 2 .
[1] B. P. S. Sahoo, C. Chou, C. Weng, et al., "Enabling millimeterwave $5 \mathrm{G}$ networks for massive IoT applications: A closer look at the issues impacting millimeter-waves in consumer devices under the $5 \mathrm{G}$ framework," IEEE Consumer Elect. Mag., vol. 8, no. 1, pp. 49-54, Jan. 2019.

[2] F. Qamar, M. N. Hindia, K. Dimyati, et al., "Investigation of future 5GIoT millimeter-wave network performance at $38 \mathrm{GHz}$ for urban microcell outdoor environment," Electronics, vol. 8, no. 495, pp. 1-26, May 2019.

[3] University of Waterloo, "Researchers develop low-power, low-cost network for 5G connectivity," https://uwaterloo.ca/math/news/researchersdevelop-low-power-low-cost-network-5g, Aug. 2019.

[4] J. Zou, H. Yu, W. Miao, et al., "Packet-based preamble design for random access in massive IoT communication systems," IEEE Access, vol. 5, pp. 11759-11767, Jun. 2017.

[5] T. Lv, Z. Lin, P. Huang, et al., "Optimization of the energy-efficient relay-based massive IoT network," IEEE Internet Things J., vol. 5, no. 4, pp. 3043-3058, Aug. 2018.

[6] R. Tian, Y. Liang, X. Tan, et al., "Overlapping user grouping in IoT oriented massive MIMO systems," IEEE Access, vol. 5, pp. 1417714186, Jul. 2017.

[7] A. Alkhateeb, O. E. Ayach, G. Leus, et al., "Channel estimation and hybrid precoding for millimeter wave cellular systems," IEEE J. Sel. Topics Signal Process., vol. 8, no. 5, pp. 831-846, Oct. 2014.

[8] J. A. Zhang, X. Huang, V. Dyadyuk, et al., "Massive hybrid antenna array for millimeter-wave cellular communications," IEEE Wireless Commun., vol. 22, no. 1, pp. 79-87, Feb. 2015.

[9] R. W. Heath Jr., N. G. Prelcic, S. Rangan, et al., "An overview of signal processing techniques for millimeter wave MIMO systems," IEEE J. Sel. Topics Signal Process., vol. 10, no. 3, pp. 436-453, Apr. 2016.

[10] A. F. Molisch, V. V. Ratnam, S. Han, et al., "Hybrid beamforming for massive MIMO: A survey," IEEE Commun. Mag., vol. 55, no. 9, pp. 134-141, Sep. 2017.

[11] S. Haghighatshoar and G. Caire, "Massive MIMO channel subspace estimation from low-dimensional projections," IEEE Trans. Signal Process., vol. 65, no. 2, pp. 303-318, Jan. 2017.

[12] P. Pal and P. P. Vaidyanathan, "Nested arrays: A novel approach to array processing with enhanced degrees of freedom," IEEE Trans. Signal Process., vol. 58, no. 8, pp. 4167-4181, Jan. 2010.

[13] A. T. Moffet, "Minimum-redundancy linear arrays," IEEE Trans. Antennas Propag., vol. 16, no. 2, pp. 172-175, Mar. 1968.

[14] E. Vertatschitsch and S. Haykin, "Nonredundant arrays," Proc. IEEE, vol. 74, no. 1, pp. 217-217, Jan. 1986.

[15] P. Pal and P. P. Vaidyanathan, "Sparse sensing with co-prime samplers and arrays," IEEE Trans. Signal Process., vol. 59, no. 2, pp. 1053-1058, Feb. 2011.

[16] L. Zhu and J. Zhu, "Optimal design of uniform circular antenna array in mmWave LOS MIMO channel," IEEE Access, vol. 6, no. c, pp. 61022-61029, Sep. 2018.

[17] P. Pal and P. P. Vaidyanathan, "Nested arrays in two dimensions, Part I: Geometrical considerations," IEEE Trans. Signal Process., vol. 60, no. 9, pp. 4694-4705, Sep. 2012.

[18] P. Pal and P. P. Vaidyanathan, "Nested arrays in two dimensions, Part II: Application in two dimensional array processing," IEEE Trans. Signal Process., vol. 60, no. 9, pp. 4706-4718, Sep. 2012.

[19] Z. Lin, T. Lv, and P. T. Mathiopoulos, "3-D indoor positioning for millimeter-Wave massive MIMO systems," IEEE Trans. Commun., vol. 66, no. 6, pp. 2472-2486, Jun. 2018.

[20] P. Forster and G. Ginolhac, "Derivation of the theoretical performance of a tensor MUSIC algorithm," Signal Process., vol. 129, no. 1, pp. 97-105, Dec. 2016.

[21] C. -L. Liu and P. P.Vaidyanathan, "Tensor music in multidimensional sparse arrays," in Proc. IEEE Asil. Conf. on Sig., Sys., and Comp., Pacific Grove, CA, USA, Nov. 2015, pp. 1783-1787.

[22] Z. Zhou, J. Fang, L. Yang, et al., "Low-rank tensor decomposition-aided channel estimation for millimeter wave MIMO-OFDM systems," IEEE J. Sel. Areas Commun., vol. 35, no. 7, pp. 1524-1538, Jul. 2017.

[23] T. Basikolo, K. Ichige, and H. Arai, "Direction of arrival estimation for quasi-stationary signals using nested circular array," in Proc. Int. Workshop Compressed Sens. Theory Appl. Radar, Sonar Remote Sens. (CoSeRa), Aachen, Germany, Sep. 2016, pp. 193-196. 
[24] T. Basikolo, K. Ichige, and H. Arai, "Nested circular array and its concentric extension for underdetermined direction of arrival estimation," IEICE Trans. Commun., vol. 101, no. 4, pp. 1084-1096, Apr. 2018.

[25] L. D. Lathauwer, B. D. Moor, and J. Vandewalle, "A multilinear singular value decomposition," SIAM J. Matrix Anal. Appl., vol. 21, no. 4, pp. 1253-1278, Mar. 2000.

[26] L. Zhao, G. Geraci, T. Yang, et al., "A tone-based AoA estimation and multiuser precoding for millimeter wave massive MIMO," IEEE Trans. Commun., vol. 65, no. 12, pp. 5209-5225, Dec. 2017.

[27] X. Shen and A. I. Zayed, Multiscale signal analysis and modeling, Springer, New York, USA, 2013

[28] C. -L. Liu and P. P.Vaidyanathan, "Hourglass arrays and other novel 2-D sparse arrays with reduced mutual coupling," IEEE Trans. Signal Process., vol. 65, no. 13, pp. 3369-3383, Jul. 2017.

[29] R. J. Mailloux, Phased Array Antenna Handbook, Artech House, USA, 2nd edition, 2005.

[30] S. Han, C. I, Z. Xu, et al., "Large-scale antenna systems with hybrid analog and digital beamforming for millimeter wave 5G," IEEE Commun. Mag., vol. 53, no. 1, pp. 186-194, Jan. 2015.

[31] D. H. Johnson and D. E. Dudgeon, Array Signal Processing: Concepts and Techniques, NJ: Prentice-Hall, Englewood Cliffs, USA, 1993.

[32] R. T. Hoctor and S. A. Kassam, "The unifying role of the coarray in aperture synthesis for coherent and incoherent imaging," Proc. IEEE, vol. 78, no. 4, pp. 735-752, Apr. 1990.

[33] M. C. Vanderveen, A. J. van der Veen, and A. Paulraj, "Estimation of multipath parameters in wireless communications," IEEE Trans. Signal Process., vol. 46, no. 3, pp. 682-690, Mar. 1998.

[34] W.-K. Ma, T.-H. Hsieh, and C.-Y. Chi, "DOA estimation of quasistationary signals via Khatri-Rao subspace," in Proc. Int. Conf. Acoust., Speech Signal Process. (ICASSP), Taipei, Taiwan, Apr. 2009, pp. 2165-2168.

[35] T. G. Kolda and B. W. Bader, "Tensor decompositions and applications," SIAM Rev., vol. 51, no. 3, pp. 455-500, Sep. 2009.

[36] H. Wang and M. Kaveh, "Coherent signal-subspace processing for the detection and estimation of angles of arrival of multiple wide-band sources," IEEE Trans. Acoust. Speech Signal Process., vol. 33, no. 4, pp. 823-831, Aug. 1985.

[37] L. N. Ribeiro, S. Schwarz, M. Rupp, et al., "Energy efficiency of mmwave massive MIMO precoding with low-resolution DACs," IEEE J. Sel. Topics Signal Process., vol. 12, no. 2, pp. 298-312, May 2018.

[38] F. Wen, N. Garcia, J. Kulmer, et al., "Tensor decomposition based beamspace ESPRIT for millimeter wave MIMO channel estimation," in Proc. IEEE Int. Global Commun. (GLOBECOM), Abu Dhabi, United Arab Emirates, Dec. 2018, pp. 1-7.

[39] D. Wang, M. Fattouche, and X. Zhan, "Pursuance of mm-level accuracy: Ranging and positioning in mmWave systems," IEEE Systems J., vol. 13, no. 2, pp. 1169-1180, Jun. 2019.

[40] G. N. Watson, A Treatise on the Theory of Bessel Functions, Cambridge Univ. Press, Cambridge, UK, 2nd edition, 1952.

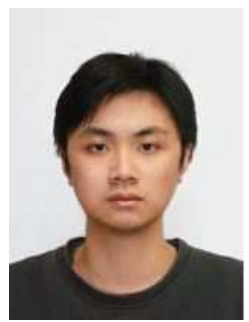

Zhipeng Lin (S'17) is currently working toward the dual Ph.D. degrees in communication and information engineering with the School of Information and Communication Engineering, Beijing University of Posts and Telecommunications, Beijing, China, and the School of Electrical and Data Engineering, University of Technology of Sydney, Sydney, NSW, Australia. His current research interests include millimeter-wave communication, massive MIMO, hybrid beamforming, wireless localization, and tensor processing.

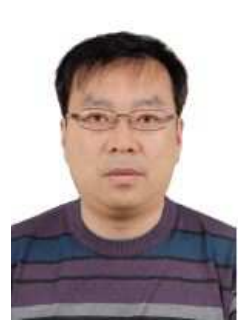

Tiejun Lv (M'08-SM'12) received the M.S. and $\mathrm{Ph} . \mathrm{D}$. degrees in electronic engineering from the University of Electronic Science and Technology of China (UESTC), Chengdu, China, in 1997 and 2000, respectively. From January 2001 to January 2003, he was a Postdoctoral Fellow with Tsinghua University, Beijing, China. In 2005, he was promoted to a Full Professor with the School of Information and Communication Engineering, Beijing University of Posts and Telecommunications (BUPT). From September 2008 to March 2009, he was a Visiting Professor with the Department of Electrical Engineering, Stanford University, Stanford, CA, USA. He is the author of 3 books, more than 80 published IEEE journal papers and 180 conference papers on the physical layer of wireless mobile communications. His current research interests include signal processing, communications theory and networking. He was the recipient of the Program for New Century Excellent Talents in University Award from the Ministry of Education, China, in 2006. He received the Nature Science Award in the Ministry of Education of China for the hierarchical cooperative communication theory and technologies in 2015.

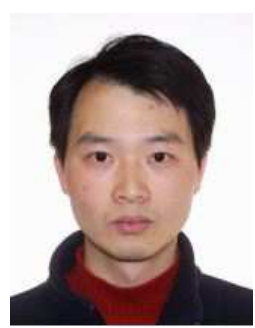

Wei Ni (M'09-SM'15) received the B.E. and Ph.D. degrees in Electronic Engineering from Fudan University, Shanghai, China, in 2000 and 2005, respectively. Currently, he is a Group Leader and Principal Research Scientist at CSIRO, Sydney, Australia, and an Adjunct Professor at the University of Technology Sydney and Honorary Professor at Macquarie University, Sydney. He was a Postdoctoral Research Fellow at Shanghai Jiaotong University from 2005 - 2008; Deputy Project Manager at the Bell Labs, Alcatel/Alcatel-Lucent from 2005 to 2008; and Senior Researcher at Devices R\&D, Nokia from 2008 to 2009. His research interests include signal processing, stochastic optimization, learning, as well as their applications to network efficiency and integrity.

$\mathrm{Dr} \mathrm{Ni}$ is the Chair of IEEE Vehicular Technology Society (VTS) New South Wales (NSW) Chapter since 2020 and an Editor of IEEE Transactions on Wireless Communications since 2018. He served first the Secretary and then Vice-Chair of IEEE NSW VTS Chapter from 2015 to 2019, Track Chair for VTC-Spring 2017, Track Co-chair for IEEE VTC-Spring 2016, Publication Chair for BodyNet 2015, and Student Travel Grant Chair for WPMC 2014.

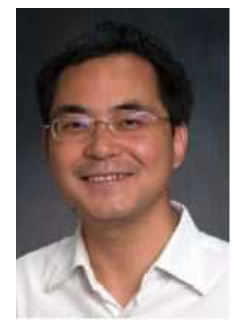

J. Andrew Zhang (M'04-SM'11) received the B.Sc. degree from Xi' an JiaoTong University, China, in 1996, the M.Sc. degree from Nanjing University of Posts and Telecommunications, China, in 1999, and the Ph.D. degree from the Australian National University, in 2004

Currently, Dr. Zhang is an Associate Professor in the School of Electrical and Data Engineering, University of Technology Sydney, Australia. He was a researcher with Data61, CSIRO, Australia from 2010 to 2016, the Networked Systems, NICTA, Australia from 2004 to 2010, and ZTE Corp., Nanjing, China from 1999 to 2001. Dr. Zhang's research interests are in the area of signal processing for wireless communications and sensing. He has published more than 180 papers in leading international Journals and conference proceedings, and has won 5 best paper awards. He is a recipient of CSIRO Chairman's Medal and the Australian Engineering Innovation Award in 2012 for exceptional research achievements in multi-gigabit wireless communications. 


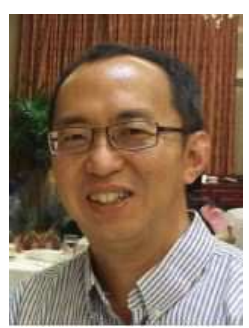

Ren Ping Liu (M'09-SM'14) received his B.E. and M.E. degrees from Beijing University of Posts and Telecommunications, China, and the Ph.D. degree from the University of Newcastle, Australia.

He is currently a Professor and Head of Discipline of Network \& Cybersecurity at University of Technology Sydney. Professor Liu was the co-founder and CTO of Ultimo Digital Technologies Pty Ltd, developing IoT and Blockchain. Prior to that he was a Principal Scientist and Research Leader at CSIRO, where he led wireless networking research activities. He specialises in system design and modelling and has delivered networking solutions to a number of government agencies and industry customers. His research interests include wireless networking, Cybersecurity, and Blockchain.

Professor Liu was the founding chair of IEEE NSW VTS Chapter and a Senior Member of IEEE. He served as Technical Program Committee chairs and Organising Committee chairs in a number of IEEE Conferences. Prof Liu was the winner of Australian Engineering Innovation Award and CSIRO Chairman medal. He has over 200 research publications. 